University of Louisville

ThinkIR: The University of Louisville's Institutional Repository

$5-2010$

\title{
Investigation of high-frequency ultrasound biomicroscopy for examining arteriopathy in hand transplant patients.
}

Emily Renee Cox 1987-

University of Louisville

Follow this and additional works at: https://ir.library.louisville.edu/etd

\section{Recommended Citation}

Cox, Emily Renee 1987-, "Investigation of high-frequency ultrasound biomicroscopy for examining arteriopathy in hand transplant patients." (2010). Electronic Theses and Dissertations. Paper 283.

https://doi.org/10.18297/etd/283

This Master's Thesis is brought to you for free and open access by ThinkIR: The University of Louisville's Institutional Repository. It has been accepted for inclusion in Electronic Theses and Dissertations by an authorized administrator of ThinkIR: The University of Louisville's Institutional Repository. This title appears here courtesy of the author, who has retained all other copyrights. For more information, please contact thinkir@louisville.edu. 


\title{
INVESTIGATION OF HIGH-FREQUENCY ULTRASOUND BIOMICROSCOPY
} FOR EXAMINING ARTERIOPATHY IN HAND TRANSPLANT PATIENTS

\author{
By \\ Emily Renee Cox \\ B.S., University of Louisville, 2009
}

\author{
A Thesis \\ Submitted to the Faculty of the \\ University of Louisville \\ J.B. Speed School of Engineering \\ in Partial Fulfillment of the Requirements \\ for the Professional Degree
}

MASTER OF ENGINEERING

Department of Bioengineering

May 2010 



\title{
INVESTIGATION OF HIGH-FREQUENCY ULTRASOUND BIOMICROSCOPY
} FOR EXAMINING ARTERIOPATHY IN HAND TRANSPLANT PATIENTS

Submitted by:

$$
\text { Emily Cox }
$$

A Thesis Approved on

\section{(Date)}

by the Following Reading and Examination Committee:

\author{
Dr. Robert Keynton, Thesis Director
}

Dr. Gail DePuy

\author{
Dr. Ayman El-Baz
}




\section{ACKNOWLEDGEMENTS}

I would like to my parents, Rick and Joan Cox, who have instilled in me a great value to my education. They have encouraged me along my educational journey, and have provided cherished support along the way. I would also like to thank my siblings, David, Jennifer, and Olivia. To all of my family and friends, I would like to express my sincere gratitude for listening to me and providing me with valuable advice in times of doubt and when the answers were not clear. A special thank you belongs to Scott Nicholas, who has provided a large amount of moral support, as well as much needed technical support throughout the year.

I would also like thank Dr. Robert Keynton who introduced this interesting project to me and for his mentorship throughout not only this year, but all five of my years spent in college. He has challenged me to perform exceptionally well and pursue excellence. I would like to thank Dr. Christina Kaufman for her help in most of the clinical aspects of this project. I would also like to thank Brenda Blair for her support with patient histories and for her patience in organizing and preparing for the patient examinations. I would like to thank Dr. Gail DePuy for her helpful advice regarding statistical analyses. And to anyone else that I accidently forgot, please know that I greatly appreciated all of your help and support. 


\begin{abstract}
The fourth hand transplant recipient in Louisville lost his graft at nine months post transplant from ischemia due to severe graft arteriopathy. Conventional imaging techniques and clinical measurements, including CT angiography and measurements of brachial indices via ultrasound, did not indicate that the patient was in such a severe condition in the days prior to amputation. Histological analysis of tissue from the amputated graft revealed massive intimal hyperplasia, a hallmark of chronic rejection. The purpose of this study was to describe a method to allow non-invasive monitoring of wall thicknesses in the radial, ulnar, palmar arch, and digital arteries of the transplanted hand.
\end{abstract}

A novel ultrasound biomicroscopy unit (Vevo 2100) was used to image the blood vessels of the transplanted and native hands in four hand transplant recipients, ranging from 16 months to 11 years post transplant. The unit has a potential resolution of $30 \mu \mathrm{m}$, which allowed measurement of the intima and media layers of the arteries.

The intima, media, and lumen measurements were compared in the native and transplanted hand in all four patients. Significant increases in intima thickness were found in the transplanted hands. In addition, the transplanted hand had significantly higher intima to lumen diameter ratios. From Doppler waveforms, it was found that that peak velocities were lower in the transplanted hand. Finally, hoop stress calculations revealed that the hoop stress seen in the arterial walls was lower in the transplanted hand.

The ultrasound biomicroscopy unit provided an accurate, repeatable, and realtime method to investigate the arteriopathy of hand transplant patients. In the future, it 
will provide a way for clinicians to monitor the progression of arteriopathy in the hand, including intimal hyperplasia. 


\section{TABLE OF CONTENTS}

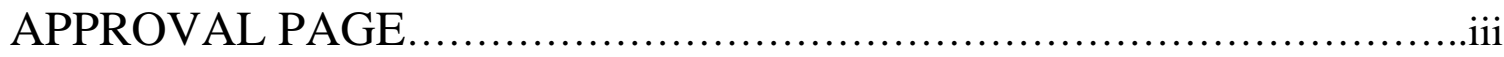

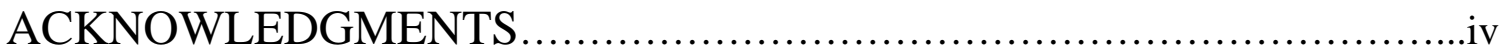

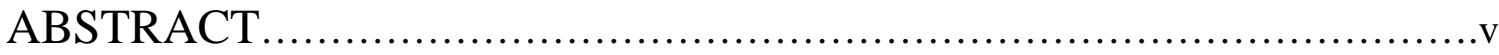

NOMENCLATURE.............................................................

LIST OF TABLES.............................................................

LIST OF FIGURES....................................................................

I. INTRODUCTION .....................................................

1.1 Purpose of the study ...........................................................

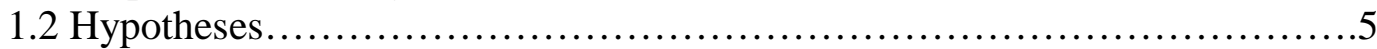

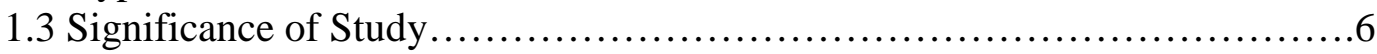

II. LITERATURE REVIEW................................................

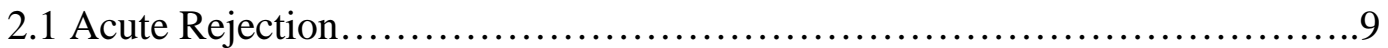

2.2 Chronic Rejection................................................. 10

2.3 Intimal Hyperplasia.................................................... 11

2.4 Current Methods to Assess Arterial Patency ..................................13

2.5 Ultrasound Biomicroscopy................................................18

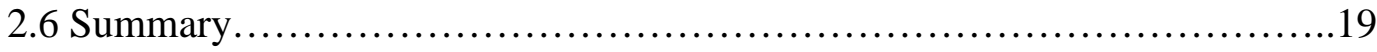

III. INSTRUMENTATION AND EQUIPMENT .........................21

3.1 Digital Brachial Indices Instrumentation..................................21

3.2 Ultrasound Biomicroscopy Instrumentation.............................22

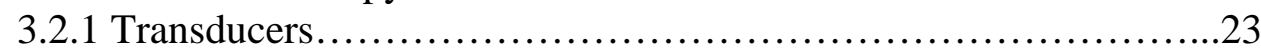

3.3 Sphygmomanometer and Stethoscope................................24

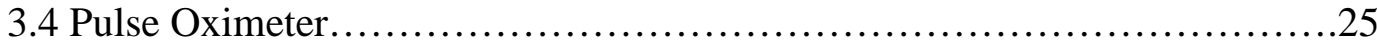

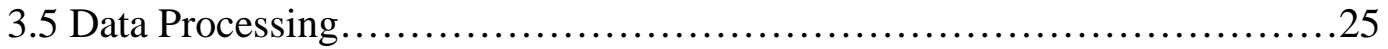

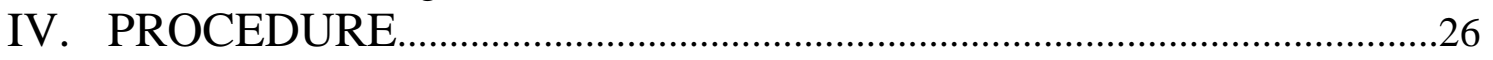

4.1 Hand Transplantation Patient Profiles.....................................26

4.2 Ultrasound for Obtaining Digital Brachial Indices..........................30

4.3 Ultrasound Biomicroscopy...................................................

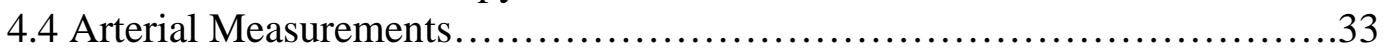

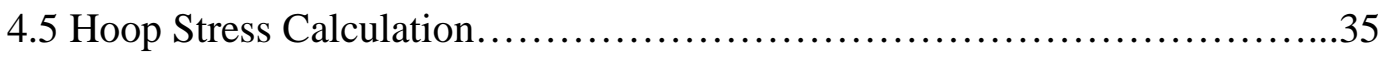

4.6 Blood Pressure Measurement...............................................

4.7 Oxygen Saturation Measurement........................................36

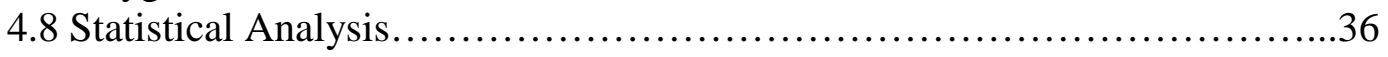

V. RESULTS AND DISCUSSION OF RESULTS...........................37 
5.1 Brachial Index Measurement..........................................37

5.2 Ultrasound Biomicroscopy Measurements.............................41

5.2.1 Intima Thickness............................................41

5.2.2 Intima-media and MediaThicknesses.............................45

5.2 .3 Lumen diameter............................................49

5.2.4 Intima to Lumen Diameter Ratio...............................51

5.2.5 Intima-media to Lumen Diameter and Media to Lumen Diameter Ratio.........................................................53

5.3 Blood Pressure Measurements........................................56

5.4 Oxygen Saturation Measurements.......................................57

5.5 Hoop Stress Calculation for Arterial Wall................................57

VI. CONCLUSIONS ...................................................62

VII. RECOMMENDATIONS ..........................................63

\section{APPENDIX I}

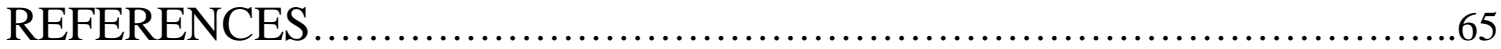

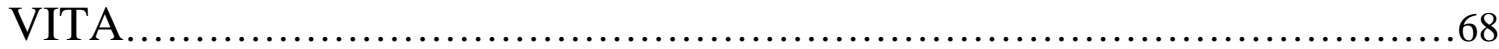




\title{
NOMENCLATURE
}

\author{
$\mathrm{CMV}=$ cytomegalovirus \\ $\mathrm{CT}$ = computed tomography \\ CTA = composite tissue allotransplantation \\ $\mathrm{ESRD}=$ end-stage renal disease \\ GVHD = graft versus host disease \\ HHS = hypothenar hammer syndrome \\ $\mathrm{IH}=$ intimal hyperplasia \\ $\mathrm{IMT}=$ intima-media thickness \\ IT = intima thickness \\ $\mathrm{LD}=$ lumen diameter \\ MMF = mycophenolate mofetil \\ MRA = magnetic resonance angiography \\ $\mathrm{MT}=$ media thickness \\ PPG = photoplethysmography \\ $\mathrm{UBM}=$ ultrasound biomicroscopy
}




\section{LIST OF TABLES}

TABLE I - VEVO 2100 TRANSDUCER CHARACTERISTICS......................23

TABLE II - ARTERIES EXAMINED ...........................................30 


\section{LIST OF FIGURES}

Figure 1 -- Histological slide of donor radial artery taken at CTA graft amputation.....18

Figure 2 -- Histological slide of donor ulnar artery taken at CTA graft amputation.......18

Figure 3 -- Flo-Lab 2100-SX for brachial index measurements.......................24

Figure 4 - The Vevo 2100 ultrasound biomicroscopy system.....................25

Figure 5 -- Moore Medical 775-660 sphygmomanometer and stethoscope..............26

Figure 6 -- Nonin Onyx 9500 pulse oximeter.................................27

Figure 7 -- Ultrasound image acquired by the UBM for a radial artery from a

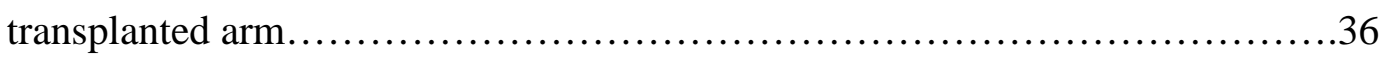

Figure 8 -- Image defining the lumen, intima, and media layers extracted from an UBM

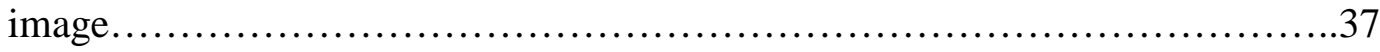

Figure 9 - Plot of brachial index vs. intima thickness..........................61

Figure 10 -- Plot of brachial index vs. IT-to-LD ..................................62

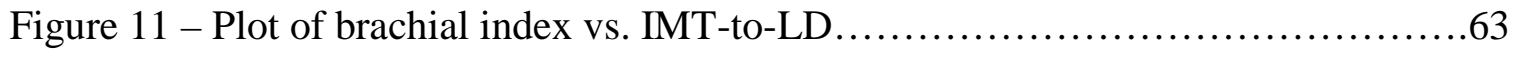




\section{INTRODUCTION}

The loss of a limb is a potentially devastating event in a person's life that often results in profound physical, psychological, and occupational consequences. There are approximately 1,908 upper limb amputations performed each year. The majority of upper limb amputations are necessary due to traumatic injury or congenital disorders (Adams, 1999).

Conventional treatment options available for upper limb amputees include: 1) reconstructive surgery using their own tissue to repair the damage; or, 2) the use of a prosthetic device. Recently, a third option, composite tissue allotransplantation (CTA), referring to the transplantation of allogenic grafts made up of more than one type of tissue (i.e., skin, muscle, and bone), has become available (Kaufman, 2009). CTA combines the principles of microsurgical reconstruction and human organ transplantation. CTA has the potential to be useful for functional restoration of patients with severe tissue loss as encountered with massive burns, traumatic injuries, congenital anomalies, and following tumor resection. Many of these patients simply do not have enough autologous tissue for the surgeon to use. For example, instead of a surgeon having the situation where he would need to reconstruct a jawbone by using bone from the tibia, the surgeon could replace "like tissue" with "like tissue" in CTA. The ability to reliably and safely carry out CTA would revolutionize reconstructive surgery.

The field of CTA transplantation is still very new. There have only been $\sim 44$ hands transplanted worldwide. The Louisville CTA program, which is a collaboration between the surgeons at Kleinert and Kutz and Associates, the Christine M. Kleinert Institute for Hand and Microsurgery, the University of Louisville, and Jewish Hospital and St. Mary’s 
Healthcare, was the first center to perform hand transplantations in the United States and have transplanted five hands, with their first transplant occurring in January 1999. Other types of CTA grafts including abdominal walls, larynx and knees have also been transplanted. Although CTA has many benefits, CTA also introduces new complications to consider in tissue reconstruction such as immunologic, ethical, and psychological issues (Petit, 2003).

Currently, recipients of CTA must receive systemic immunosuppressive treatment for the life of the graft, which has potentially serious side effects. Finding an effective and nontoxic immunosuppressive treatment has been a limiting factor in organ transplantation. Composite tissue allografts, unlike solid organ transplants, are heterogeneous and are composed of tissues that express varying degrees of antigenicity. Thus, increasing antigenic sensitivity of CTA complicates the development of an ideal immunosuppressive treatment further (Petit, 2003).

Allograft patients are at risk for acute and chronic graft rejection, immunosuppressive toxicity, and graft-versus-host disease (GVHD). Physicians use several methods to check for signs and symptoms of rejection. In particular, they look for arteriopathy that could threaten the blood flow and viability to the graft. Signs of rejection are first suspected upon visual inspection of the transplanted hand. Then, other diagnostic measures, such as brachial indices, histology, CT, and MR angiography, are used in order to confirm or disprove the suspicion. The gold standard for diagnosing rejection in CTA is histological evaluation of skin biopsies (Cendales 2007). Due to the invasiveness of taking a biopsy for histological evaluation, the other mentioned diagnostic measures are performed first. 
Physicians use brachial indices to evaluate vascular perfusion of the hand and digits in several conditions such as rheumatoid arthritis, systemic lupus, Berger's disease, atherosclerosis, and trauma. In each of these conditions, vascular supply is decreased. The brachial indices are obtained from an ultrasound that measures segmental blood pressure of the arteries in the arm and hand. For hand transplant, digital brachial indices are used to eliminate the suspicion of any obstructive phenomenon which may compromise perfusion. This could include an anastomosic site with developing stenosis or advanced intimal hyperplasia. The brachial indices are pressure ratios, which may not provide much valuable information regarding the patency of the vessels investigated.

$\mathrm{CT}$ and MR angiography are noninvasive imaging techniques that provide a visualization of the arterial tree to assess patency of the blood vessels in the graft. However, only the late stages of chronic rejection would show observable differences in the patency of these arteries. To determine the status of the graft, it is important to examine the arteriopathy present. In transplants, arteriopathy generally manifests as cellular growth in the arterial wall. The arterial wall is only several micrometers thick. Thus, to detect the arteriopathy present in hand transplant patients, the resolution of an imaging technique must be on the micrometer range. The resolution of both CT and MR angiography is not sufficient to image the arterial wall; and, therefore, it cannot be used to detect the arteriopathy present in hand transplant patients.

Arterial intimal hyperplasia (IH) refers to any cells that form layers between the endothelial lining and internal elastic membrane of the arterial wall. Arterial neointimal formation or intimal hyperplasia is consistently present in graft vascular disease (Subbotin, 2007). In addition, it is the main manifestation of chronic rejection in solid 
organ transplantation (Demetris, 1998; Geraghty, 1996). Progression of intimal hyperplasia can lead to occluded arteries or stenosis of vascular grafts (Subbotin, 2007; Demetris, 1998; Geraghty, 1996; Haruguchi, 2003).

The exact pathology of intimal hyperplasia remains unclear. There are a number of factors that are suspected to either initiate or aggravate the progression of intimal thickening. Intimal hyperplasia is known to develop distal to arterial bypass graft anastomoses. Several mechanical factors including low shear stress, oscillating shear forces, high wall shear stress gradients, and compliance mismatch have been attributed to intimal hyperplasia formation (Haruguchi, 2003; Kohler, 1992; Glagov, 1997; Keynton, 1995). For transplant patients, the introduction of alloantigens and an activated immune system are thought to be the underlying mechanism of graft vascular disease, which presents itself as intimal hyperplasia (Subbotin, 2007).

Monitoring the progression of intimal hyperplasia in the past has been difficult. However, this method has several drawbacks, most importantly, unacceptable morbidity at the biopsy site. This is especially true for deep biopsies. A patient can undergo deep biopsy a limited number of times since the procedure permanently removes a section of the arterial tree. In addition, a histological analysis of an arbitrary arterial slice may not provide an accurate representation of the level of arteriopathy throughout the same artery longitudinally. Intimal hyperplasia can be patchy, meaning that it may be thicker in some areas and much thinner in the same artery just a few centimeters away. Finally, a biopsy does not give a real-time indication of the level of arteriopathy. The tissue sample excised must be sent to a laboratory for analysis, and the patient must wait for the results. 
Thus, identifying or developing a new, non-invasive, high resolution system for measuring intimal hyperplasia thickness in arterial vessels would significantly impact the field of CTA. A system with these capabilities would enable the clinician to carefully monitor the progression of the IH post-operatively. As a result, the clinician could properly prescribe or modify the therapeutic regimen to retard, stop or reduce the growth of $\mathrm{IH}$ in the transplanted graft.

\subsection{Purpose of the Study}

The purpose of this study was to identify and characterize a repeatable, real-time, non-invasive method, Ultrasound Biomicroscopy (UBM) system (Vevo 2100, Visualsonics, Toronto, Canada) to measure intimal hyperplasia in CTA grafted patients and compare levels of intimal hyperplasia between the native and transplanted hands. In addition, measurements from the UBM are to be compared with the digital brachial index measurements to investigate the validity of using digital brachial indices as an indirect assessment of arterial patency.

\subsection{Hypotheses}

1) The intima thicknesses are significantly higher in the transplanted arm than in the native arm in hand transplant patients, using the patient as his own control.

2) The intima-to-lumen diameter ratios are significantly higher in the arm receiving the CTA graft than in the native arm of hand transplant patients. 
3) Intima thickness and lumen diameter more accurately indicate the presence of arteriopathy compared to brachial indices, the current gold standard.

4) Brachial indices do not directly correlate to CTA arteriopathy.

\subsection{Significance of Study}

Chronic rejection is a poorly understood phenomenon and difficult to monitor. In previous studies, chronic rejection was not considered to be a significant problem in CTA (Kaufman, 2009; Petit, 2003; Petruzzo, 2008). However, recent event in our group have indicated that chronic rejection via excessive hyperplastic growth of the intima does occur in CTA graft patients. Conventional vascular imaging techniques are unable to detect arteriopathy. Thus, a suitable method to detect and observe changes in the arterial wall will provide clinicians with the opportunity to detect chronic rejection in composite tissue allotransplantation early, which will enable the clinicians to modify the patient's therapeutic regimen in order to halt or slow the progression of the threatening arteriopathy. In addition, a safe and non-invasive intimal hyperplasia measurement technique can provide a means to better understand what normal levels of intimal hyperplasia are and help in the identification of risk factors involved in IH development. 


\section{LITERATURE REVIEW AND BACKGROUND}

There are approximately 1.7 million people living with limb loss in the United States (Adams, 1999). There is a much smaller group of upper-limb amputees, given that approximately 1, 908 upper-limb amputations are performed each year compared to 56,912 lower-limb amputations. The majority of all trauma-related amputations (about 68.6 percent) are upper-limb amputations. Risk of traumatic amputations increase with age and is higher for men than women (NLLIC, 2008).

Treatment options available for individuals that have lost a hand or limb include reconstructive surgery using their own tissue to repair the damage or the use of a prosthetic device. Recently, another option has been introduced clinically, composite tissue allotransplantation (CTA). Referring to all non-organ transplants, CTA combines the principles of microsurgical reconstruction and human organ transplantation. The transplantation tissue for repairing the lost limb is taken from deceased donors that have also donated their hearts, kidneys or other types of organs to carefully selected patients (Petit, 2003). CTA aims to address the limitations associated with reconstructive surgery by using native tissue, including poor functional and aesthetic results, multiple revisions, long rehabilitation times, and donor site morbidity (Breidenbach, 2008). However, CTA also introduces new complexities to consider in tissue reconstruction such as immunologic, ethical, and psychological issues (Petit, 2003).

Allograft patients are at risk for graft-versus-host disease (GVHD), immunosuppressive toxicity, and acute and chronic rejection. To prevent these conditions, the recipient of a CTA graft must comply with an immunosuppressive 
protocol indefinitely, which has potentially serious side effects. The search for an efficient yet nontoxic immunosuppressive treatment has been the primary limitation in the field of transplantation. Unlike solid organ transplants, composite tissue allografts are histologically heterogeneous and are composed of tissues that express varying degrees of antigenicity, such as skin and muscle, which are extremely antigenic. The greater antigenicity of composite tissues further complicates the development of desirable immunosuppression regimens (Petit, 2003).

GVHD occurs when donor T cells respond to protein on host cells that are genetically specific to the host (Ferrara, 2009). The principal proteins involved are the human leucocyte antigens (HLAs), which are highly polymorphic and are encoded by the major histocompatibility complex (MHC). Class I HLA (A, B, and C) proteins are expressed on nearly all nucleated cells of the body. Class II proteins (DR, DQ, and DP) are mainly expressed on haemopoietic cells (B cells, dendritic cells, and monocytes), but their expression can be induced on several other cell types after inflammation or injury. The frequency of acute GVHD is directly correlated to the degree of mismatch between HLA proteins.

Typically, a triple-drug combination therapy has been used for CTA immunosuppression, similar to the standard approach used in solid organ transplantation. Each of the hand transplants reported to the International Registry on Hand and Composite Tissue Transplantation (IRHCTT) before 2009 utilized a combination of tacrolimus, mycophenolate mofetil (MMF), and a steroid (usually prednisone) in the first period after transplantation. The patients were usually weaned-off of the steroids, if possible, to reduce severity of the side effects. 
The immunosuppressive drugs that CTA patients take to protect the viability of their graft act nonspecifically to suppress the entire immune system (Ravindra, 2009). This results in an associated risk of tumors, opportunistic infections, and organ toxicity. The hand transplant registry reports opportunistic infections in 63.6\% and metabolic complications in $50 \%$ of the patients. The most serious complications that have been reported have been steroid-related aseptic necrosis of both hips in a patient (Gonzalez, 2005) and renal compromise in the first face transplant recipient (Dubernard, 2007). The main challenges for CTA are minimizing immunosuppression while also preventing acute and chronic rejection (Ravindra, 2009).

Both acute and chronic rejection episodes are first suspected upon visible signs on the skin of the transplanted hand, this typically manifests as a maculopapular rash (flat red area on the skin covered with small confluent bumps). Rejection episodes are then confirmed by histological evaluation of a skin biopsy (Breidenbach, 2008). Acute rejection episodes occur in some degree in all transplants. However, recurrent episodes may be associated with a more severe condition, chronic rejection. Chronic rejection is the process of long-term loss of function of the transplant. Care must be taken to watch for signs of both acute and chronic rejection. A large amount of research has been dedicated to the signs, symptoms, and treatments for acute and chronic rejection.

\section{$\underline{2.1 \text { Acute Rejection }}$}

Acute rejection is a T cell-mediated attack of the allograft by the recipient's immune system. Acute rejection results in graft damage and loss of the graft if not promptly treated with a more aggressive immunosuppression therapy. Episodes of acute 
rejection are characterized by cutaneous lesions, which generally begin as faint pink macules that may develop into red infiltrated papules with or without swelling of the graft. The majority of acute rejection episodes occur in the first year after transplantation. Acute rejection episodes occurred in about $85 \%$ of the IRHCTT patients within the first year; many patients had multiple acute rejection episodes in this first year post-transplant. These episodes were easily reversed with an increase of immunosuppression (Ferrara, 2009). The rejection episodes were reversed in all compliant hand transplant patients when the episodes were promptly reported and treated (Petruzzo, 2008). Other acute rejection episodes occurred when patients did not comply with their immunosuppressive drug regimen or when their drug therapy was reduced for various reasons (i.e., to reduce side effects or as a team decision) (Petruzzo, 2008).

\subsection{Chronic Rejection}

Chronic rejection has remained a poorly characterized event that usually occurs late after transplantation. Classical features of chronic rejection that differentiate it from acute rejection are intimal hyperplasia and interstitial fibrosis (Petruzzo, 2008). Chronic rejection mechanisms have been shown to be both cellular- and humoral-mediated that manifest by vascular changes (Breidenbach, 2008). Clinical and experimental studies have identified pathological markers characteristic of chronic rejection in solid organ transplantation (Geraghty, 1996; Merville, 2005; Hayry, 1991; Ross, 1993). The common factor in these studies, which marks the defining feature of chronic rejection, was the progressive thickening of the arterial walls within the affected organ due to neointimal hyperplasia. For example, in chronic renal allograft dysfunction (CRAD), the 
vascular injuries were characterized by thickening of the arterial walls with a subintimal accumulation of connective tissue, a penetration of mononuclear cells and a proliferation of myofibroblasts. These events lead to a dangerous narrowing of the arterial lumen (Merville, 2005).

Chronic rejection still disastrously affects the outcome of transplantation and there is no suitable treatment to stop it. The main pathology of chronic rejection, graft vascular disease (which often presents as intimal hyperplasia), is thought to be caused by the introduction of alloantigens and an activated immune system. Non-immunological factors have been considered to be exacerbating and possibly predictive of graft vascular disease. However, these factors have not been considered as a pathogenic cause of chronic rejection (Subbotin, 2007).

The underlying mechanism in the development of intimal hyperplasia has not been fully understood. A common theory has been that vascular smooth muscle cells from the media of the arterial wall migrate into the intima. The cause for this potential mechanism has remained unknown. Scientists have theorized that it could be immunologically and/or mechanically mediated. Very few studies have performed experiments to confirm or dismiss this theory because of the inherent difficulty in assessing the progression of intimal hyperplasia, which produces vascular wall changes on the order of 10-100s of micrometers, in a functioning transplant (Geraghty, 1996).

\subsection{Intimal hyperplasia}

Arterial intimal hyperplasia (IH) refers to cells that form a multi-layer compartment within the internal elastic membrane of the arterial wall (in the intima layer) 
and express alpha-smooth-muscle actin (Stary, 1992: Subbotin, 2007). The thickened arterial wall results in a narrowing of the lumen diameter, which changes the flow patterns within the artery and eventually leads to the occlusion of the arterial vessel by thrombosis (Geraghty, 1996). IH is the main pathological remodeling found in complications after angioplasty, bypass operations and stenting; and once initiated, it is not treatable (Subbotin, 2007; Demetris, 1998; Geraghty, 1996; Haruguchi, 2003). In addition, IH can negatively affect other disease conditions such as peripheral artery occlusive disorder and graft vascular disease in transplantation (Subbotin, 2007; Haruguchi, 2003). Small levels of intimal hyperplasia are normal and it progresses with age; however, it rarely develops into a disease that threatens the blood supply (Subbotin, 2007).

Studies have shown that stenosis at the anastomosis of autologous and allogenic vascular grafts caused by IH may be initiated by hemodynamic changes that occur near the anastomosis (Haruguchi, 2003; Kohler, 1992; Keynton, 1995). With end-to-side anastomoses, $\mathrm{IH}$ tends to develop at the arterial floor and the toe and heel of the distal anastomosis (Haruguchi, 2003; Keynton, 1995). Low shear stress and oscillating shear index are hemodynamic factors that correlate with IH formation on the floor (Haruguchi, 2003; Keynton, 2001).

It has also been theorized that intimal thickening is a physiological adaptation to changing hemodynamic demands (Subbotin, 2007). Asakura et al. confirmed the "slowflow" effects on the development of IH by performing experiments on transparent arterial models, which maintained the true arterial geometry, where slow or disturbed flows were found to be associated with severe hyperplasia (Asakura, 1990). In vivo experiments 
using arterial bypass grafts in a dog model found that low shear stress and oscillating wall shear seem to provide a stimulus for the development of IH near the anastomosis (Keynton, 2001). It has been well-documented that aging also alters hemodynamic parameters. In regards to this fact, Osika et al. (2007) found in measurements the intima and intima-media thicknesses in radial and anterior tibial arteries increase with aging. In addition, the anterior tibial artery showed thicker intima and a thinner media compared to the radial artery, especially in the older subjects. In order to consider the different luminal diameters between the two arteries, the authors compared the intima thickness (IT) to lumen ratio and found a significant difference across all age groups investigated (ages 10-90 years) (Osika, 2007). These results may be due to the postulated "slowflow" effects that Asakura and Keynton, et al. investigated since the blood velocity in the anterior tibial artery is much lower than in the radial artery.

\subsection{Current methods to assess arterial patency}

Physicians utilize brachial indices, CT, and MR angiography to assess the performance of the grafts in hand transplant patients on a regular basis. Ultrasound is used to obtain segmental pressure measurements of arm and digital arteries. From these measurements, physicians use brachial indices to evaluate vascular perfusion of the hand and digits in several conditions such as rheumatoid arthritis, systemic lupus, Berger's disease, atherosclerosis, trauma, and hand transplantation. In each of these conditions, vascular supply is decreased. For hand transplants, digital brachial indices are used to eliminate the suspicion of any obstructive phenomena which could compromise 
perfusion. This could include an anastomosis site with developing constriction or advanced intimal hyperplasia.

A digital brachial index is obtained by calculating the ratio of the specified digital blood pressure to the simultaneous brachial artery pressure. Typically, brachial indices are also calculated for the ulnar, radial, and palmar arch arteries. Without sufficient blood flow to the artery, the surrounding tissues will not receive the oxygen and the proper nutrients needed to stay viable. In a hand transplant patient, an inadequate blood supply to the graft will lead to ischemia and eventually to rejection of the transplanted hand. Clinical practice regards brachial indices of 0.7 or less as an indication of inadequate blood flow to the particular artery. Conversely, a digital brachial index above 0.7 is indicative of adequate blood flow (Zimmerman, 1994). It is unclear why the clinically-accepted threshold has been set to 0.7 ; however, it was likely influenced by the study of Gelberman et al. (1978) and Gross et al. (1978). Gelberman studied a group of patients with replanted digits, and observed that all patients with a digital pulse pressure that was less than $75 \%$ of the contralateral side suffered from severe cold intolerance (Gelberman, 1978). While Gross et al. reported that patients with a segmental pressure of $63 \%$ or less on the contralateral side suffered from forearm claudication (Gross, 1978). Zimmerman et al. (1994) used 0.7 as a midway between these two studies to guide their choice between ligation or reconstruction of the ulnar artery in patients with ulnar artery occlusion.

MR and CT angiography are non-invasive imaging techniques that provide a visualization of the arterial tree. They allow an assessment of patency. However, the resolution of these imaging techniques is not sufficient to detect the arteriopathy that is 
present in hand transplant patients, including dangerous levels of IH. Thus, the efficiency and accuracy of these techniques for evaluation of hand transplant is questioned.

These techniques are used to evaluate the performance of transplanted hands. If a clinician discovers signs of rejection from any of these methods, he may choose to alter immunosuppression in order to halt the progress of chronic rejection. Despite these methods, the fourth hand transplant recipient in the Louisville CTA program lost his graft nine months after transplantation from ischemia due to severe graft arteriopathy. The transplant procedure performed in the Louisville CTA Program occurred in July 2008 and consisted of a short, transradial right hand transplant. The patient's post-operative therapeutic regimen consisted of an immunosuppression induction treatment (Campath 1H) which was designed to need only two types of immunosuppression. The patient was never started on steroids and was maintained on a double therapy of tacrolimus and mycophenolate mofetil (MMF). Nine months post-transplantation, this patient had unmanageable ischemia in the hand which developed over a few weeks. After failed attempts to surgically restore blood flow, the decision was made to amputate.

Histological analysis of tissue from the amputated graft indicated that ischemia was secondary to severe chronic rejection, which manifested as massive intimal hyperplasia that restricted the arteries of the allograft. The intimal hyperplasia was so severe that the lumen was miniscule. In addition, some histological slices showed thrombus formation (Figure 1 and 2). This was the first report of chronic rejection in a compliant CTA hand recipient.

Conventional imaging techniques and clinical measurements, including CT and MR angiography and measurements of digital brachial indices via ultrasound, did not 
indicate the severity of the patient's condition. Specifically, the CT angiography measurements acquired at the patient's home hospital indicated patent vessels with the only stenosis noted (judged to be 50\%) being at the anastomosic site. Similarly, a few days before amputation of the graft, the patient's transplant (right hand) radial and ulnar arteries at the wrist had brachial indices of 0.76 and 0.70 , respectively, while his native (left hand) radial and ulnar arteries at the wrist had brachial indices of 1.03 and 1.04, respectively. With these values, this patient was considered to be at or near border line since a value below 0.70 is clinically regarded as a compromise of perfusion. Analysis of punch skin biopsies two days before the graft was amputated had a histology grade of 0 , which was interpreted as no rejection (Cendales, 2007). It is important to note that the vessels investigated were not pressurized prior to histological fixation. Thus, the resulting images may provide an inaccurate indication of the severity. However, the vessels were occluded enough to warrant amputation.

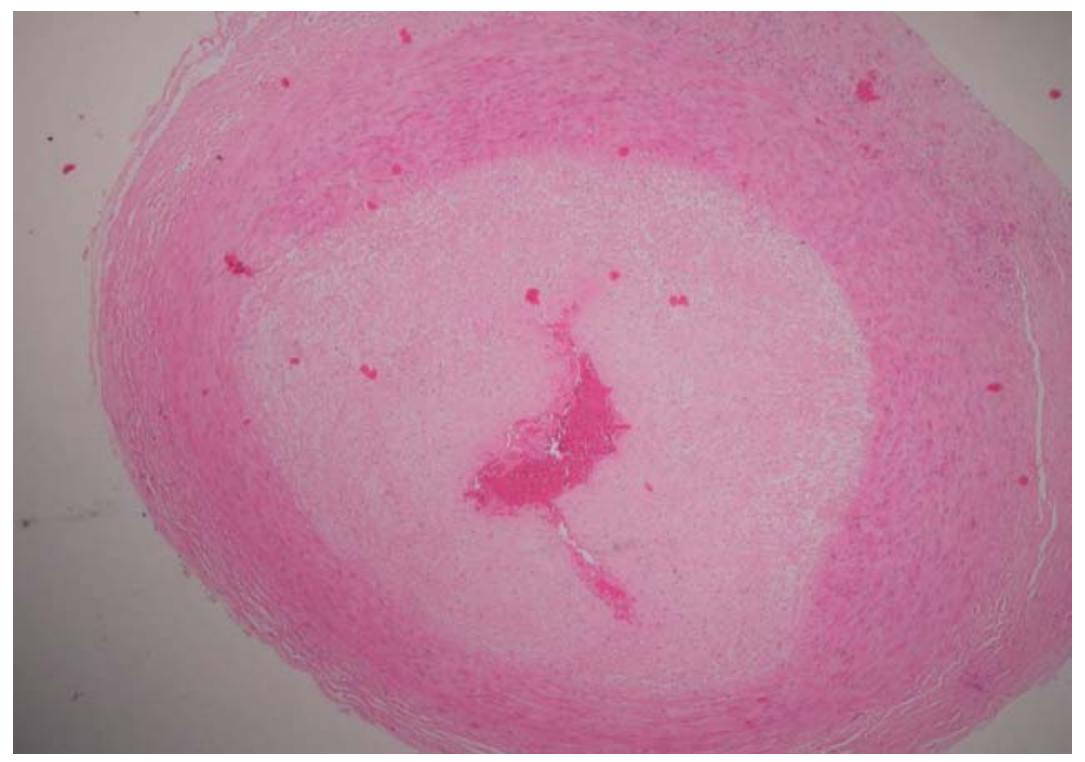

FIGURE 1 - Histological slide of donor radial artery taken at CTA graft amputation. Severe intimal hyperplasia, thrombus, and near occlusion of the lumen were noted. 


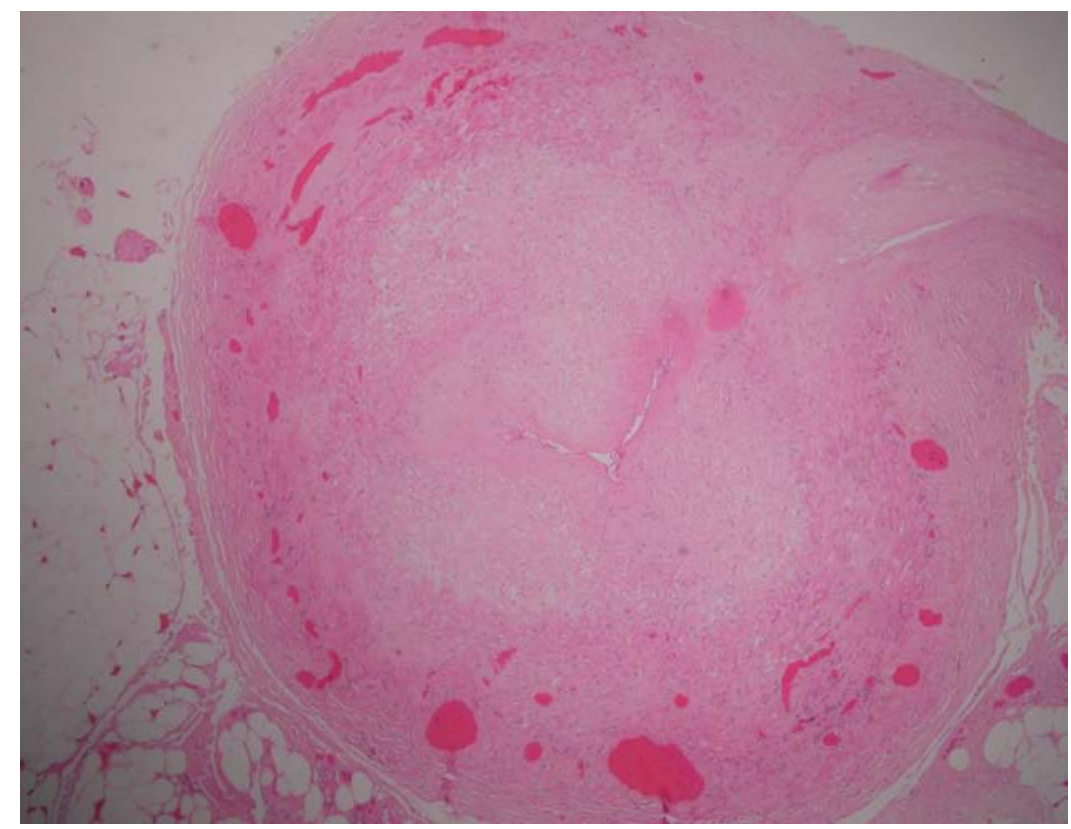

FIGURE 2 - Histological slide of donor ulnar artery taken at CTA graft amputation.

Severe intimal hyperplasia and near occlusion of the lumen were noted.

After the loss of the graft in the fourth Louisville CTA patient, the remaining four transplant patients were brought back to Louisville to look for evidence of arteriopathy and/or chronic rejection. Conventional vascular studies and punch skin biopsies of the remaining patients did not show any evidence of chronic rejection. However, deep biopsies revealed minimal to moderate arteriopathy in each of the four patients, who had no other serious indications of graft rejection. In particular, each patient revealed intimal hyperplasia. Thus, vessel wall thickening of the arterial tree should be examined closely in CTA recipients as the arterial tree may be a target of chronic rejection. The hand transplant patients that still have viable grafts have intima thicknesses between one cell layer thick (approximately $8 \mu \mathrm{m}$ ) to $0.26 \mathrm{~mm}$. Measuring these very small layers has not been possible without a biopsy accompanied with a histological examination. 


\section{$\underline{2.5 \text { Ultrasound Biomicroscopy }}$}

Until recently, only post-mortem methods of IH assessment were available via histology. However, histological preparation causes tissue shrinkage, which can significantly alter the measurement of luminal diameter changes (Razevaev, 2008). In addition, a real-time, noninvasive method is highly desirable for clinical applications to monitor the progression of vascular wall changes which impact the outcome of several diseases, including arteriosclerosis and chronic rejection. Originally developed for small animal applications, a very high resolution ultrasound system, called ultrasound biomicroscopy (UBM), was implemented to non-invasively detect in vivo structures as small as $30 \mu \mathrm{m}$.

Recently, UBM has been used in clinical studies for machine validation purposes as well as for detecting changes in the arterial walls. Razuvaev et al. (2008) demonstrated the possibility of using UBM to measure the intimal thickness in the rat carotid artery after balloon injury and to monitor the formation and progression of intimal hyperplasia during pharmacological treatment. Their UBM measurements of the intimamedia thickness in left and right common carotid artery of 15 rats correlated significantly with histological measurements ( $\mathrm{p}<0.0001)$. They also discovered that as intima-media thicknesses increased over time, lumen diameters decreased. The use of UBM to measure lumen diameter in vivo and in real time provides a new way to study arterial remodeling and vessel responses to flow changes in the arterial vessel wall (Razuevaev, 2008).

Osika et al. (2007) utilized UBM to determine the thickness of artificial layers of silicone and intima thickness (IT) of radial and anterior tibial arterires in healthy subjects 
and in patients with vascular disease. They found that IT and the IT-to-lumen ratio progresses with age. The anterior tibial artery exhibited thicker intima and thinner media thicknesses than the radial artery. In addition, the radial artery IT in patients with peripheral artery disease was thicker compared with the healthy controls. As a validation experiment and to assess UBM resolution, Osika et al. (2007) imaged silicone layer phantoms at a transducer frequency of $55 \mathrm{MHz}$. These measurements were compared to the gold standard method of measurement by weighing. They obtained a correlation coefficient of 0.98 and found a mean difference and standard deviation as low of $3.5 \pm$ $8.8 \mu \mathrm{m}$, illustrating the high accuracy associated with UBM (Osika, 2007).

Johansson et al. (2010) used the high resolution ultrasound to compare healthy subjects to those with end-stage renal disease (ESRD). They found that the IT, intimamedia thickness (IMT), and media thickness (MT) of the radial artery were all significantly higher in ESRD patients compared to healthy subjects $(\mathrm{p}<0.05)$. They also discovered that ESRD patients had significantly higher IT to lumen diameter ratios that healthy subjects $(\mathrm{p}<0.01)$. The authors speculate that this may reflect global atherosclerosis (Johansson, 2010).

\subsection{Summary}

The signs and symptoms of acute and chronic rejection have been presented. The hallmark characteristic of chronic rejection, intimal hyperplasia, is a major concern for the Louisville CTA program. Current diagnostic techniques are unable to non-invasively image or measure the intimal layer thickness of the arterial wall. Only post-mortem methods of assessing IH thickness are available via histology. Recently, a very high 
resolution ultrasound system, called ultrasound biomicroscopy (UBM), has been implemented to non-invasively detect in vivo structures as small as $30 \mu \mathrm{m}$. It provides an accurate, real-time, and non-invasive method to image the arteriopathy present in hand transplant patients.

The hand transplant clinicians at the Louisville CTA program had several questions in relation to the rejection of their fourth patient. First, they asked how this severe intimal hyperplasia manifested. They wondered if it could be induced mechanically (i.e. applying forces repetitively to the arm) or from the lower immunosuppressive regimen that was administered to this patient. Secondly, they needed a more reliable way of visualizing the transplant arteries to check for and monitor any signs of severe intimal hyperplasia or stenosis. Ideally, this method would avoid the morbidity and impracticality of multiple deep tissue biopsies. Finally, it is unknown what level of intimal hyperplasia would warrant a change in immunosuppression therapy in order to protect the graft from ischemia. Thus, identification or development of a new, non-invasive, high-resolution imaging technique capable of monitoring the progression of IH in CTA graft patients would enable clinicians to appropriately address these questions. 


\section{INSTRUMENTATION AND EQUIPMENT}

The following section contains information about instrumentation and equipment used in the completion of this thesis.

\subsection{Digital brachial indices instrumentation}

A Flo-Lab 2100-SX (Parks Medical Electronics, Aloha, OR) was used to obtain the pulse pressures in the arteries of both arms (Figure 3). The Flo-Lab system consisted of cuff inflators, Doppler probes, photoplethysmography (PPG) sensors, and a monitor for real-time readings. From there, the digital brachial indices were calculated. Several inflatable cuffs accompanied the machine. Large cuffs were used to find pulse pressures in the upper arm. Medium and small cuffs were used on the forearm and wrist, respectively, while very small cuffs were used to determine pulse pressure in the digits. Each cuff had a maximum pressure of $300 \mathrm{mmHg}$. A Doppler pencil probe operating at a continuous wave frequency of $8 \mathrm{MHz}$ employs sound waves and sonar detection to detect the moment when blood flow returns as the cuff is deflating. Then, it is able to gather the pressure measurement and generate pressure waveforms for each artery, except the digital arteries. The pressure waveforms were used to gather pressure measurements of each artery. From the pressure measurements, the brachial indices were calculated. A photoplethysmography (PPG) sensor was used in the place of the Doppler probe for the digital arteries to acquire pressure data from the digital arteries by determining the moment when blood flow returns to the cuff. 


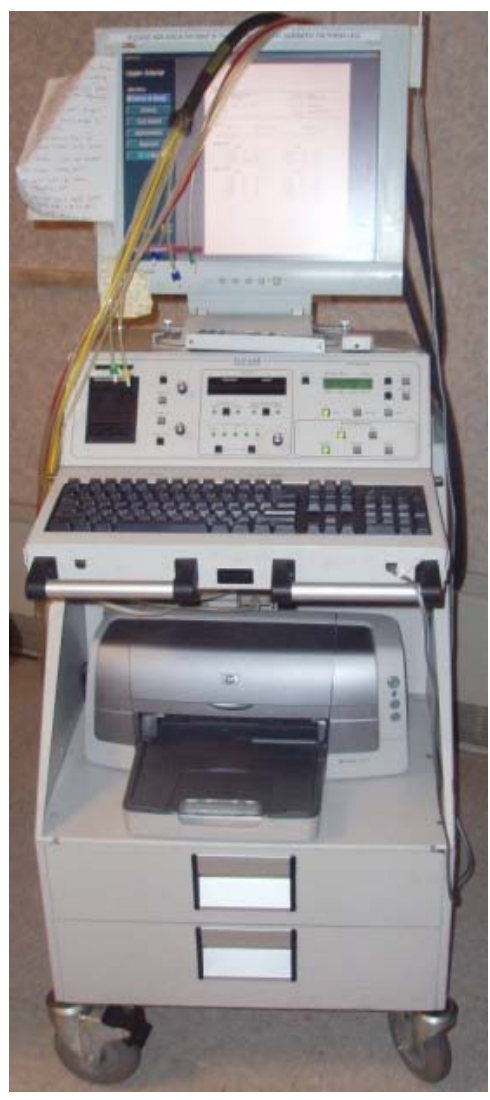

FIGURE 3 -- Flo-Lab 2100-SX for brachial index measurements

\subsection{Ultrasound Biomicroscopy Instrumentation}

The native and transplanted arteries were examined by Ultrasound Biomicroscopy (UBM; Vevo 2100, VisualSonics, Toronto, Canada) using multiple transducers with varying frequencies. Transducer selection was based on depth of the vessel to be imaged in combination with image resolution to acquire optimum intimal hyperplasia

measurements. The axial image resolution ranged from $30 \mu \mathrm{m}$ to $75 \mu \mathrm{m}$. The mainframe used a 120V, 50/60Hz AC power supply. 


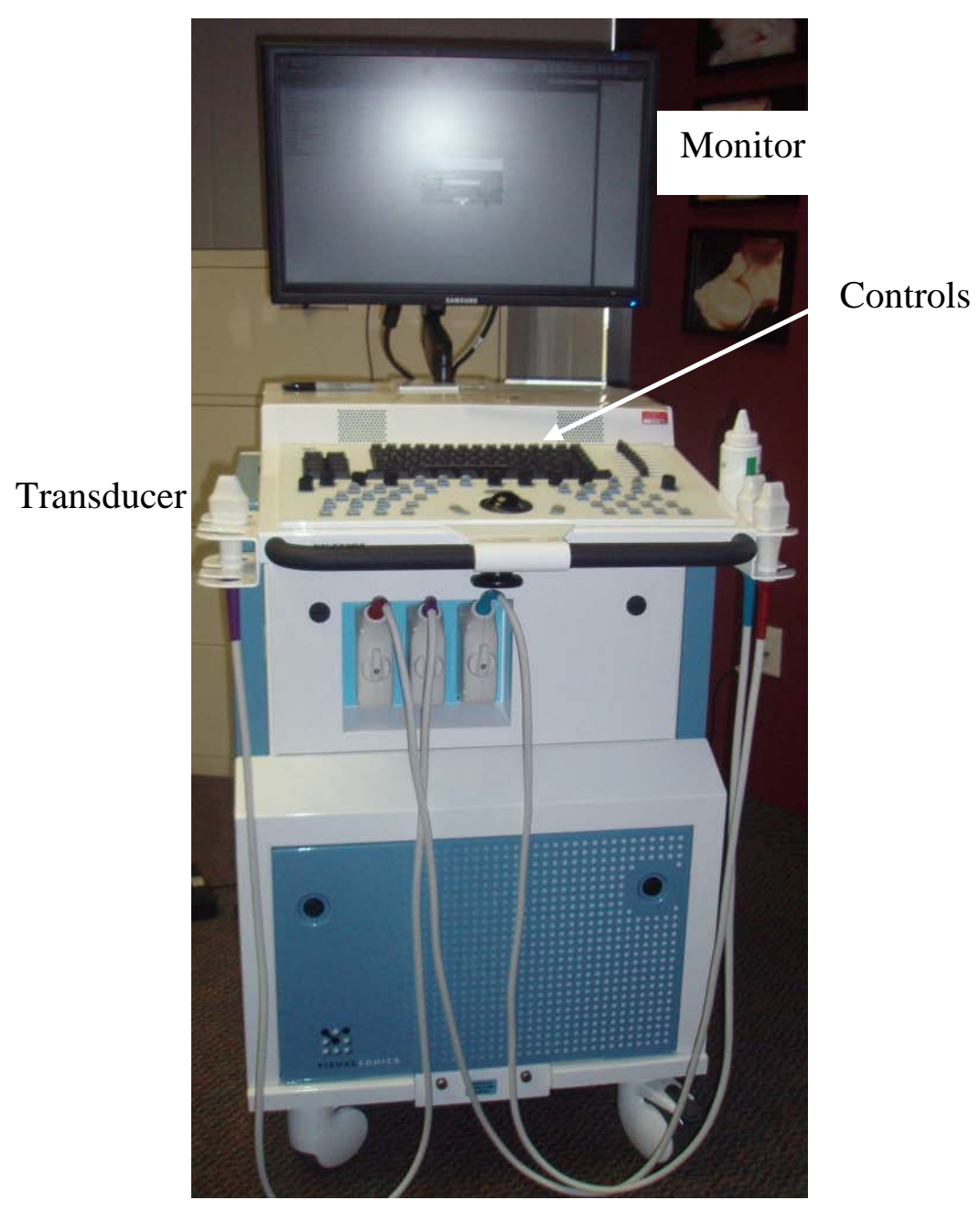

FIGURE 4 - The Vevo 2100 ultrasound biomicroscopy system.

\section{$\underline{\text { 3.2.1 Transducers }}$}

Five different transducers (MS-250, MS-400, MS-550D, MS-550S and MS-700; VisualSonics, Toronto, Canada) were used to acquire images along a line perpendicular to the vessel wall. The following table lists their center frequency, broadband frequency, geometric focus, maximum image width, maximum image depth, image axial resolution, and image lateral resolution. The resolutions were quoted at the geometric focus. 
TABLE I

VEVO 2100 TRANSDUCER CHARACTERISTICS

\begin{tabular}{|c|c|c|c|c|c|}
\hline Transducer Name & MS-250 & MS-400 & MS-550D & MS-550S & MS-700 \\
\hline Center Frequency $(\mathrm{MHz})$ & 20 & 30 & 40 & 40 & 50 \\
\hline Broadband Frequency $(\mathrm{MHz})$ & $13-24$ & $18-38$ & $22-55$ & $32-56$ & $30-70$ \\
\hline Geometric Focus $(\mathrm{mm})$ & 15 & 9 & 7 & 6 & 5 \\
\hline Max Image Width $(\mathrm{mm})$ & 23.1 & 15.4 & 14.1 & 15.1 & 9.7 \\
\hline Max Image Depth $(\mathrm{mm})$ & 36 & 20 & 15 & 15 & TBD \\
\hline Image Axial Resolution $(\mu \mathrm{m})$ & 75 & 50 & 40 & 40 & 30 \\
\hline Image Lateral Resolution $(\mu \mathrm{m})$ & 165 & 110 & 80 & 80 & 65 \\
\hline
\end{tabular}

\subsection{Sphygmomanometer and Stethoscope}

The blood pressure of each subject was measured before and after UBM

examination. The blood pressure measurements were obtained using a Moore Medical 775-660 (New Britain, CT) professional aneroid sphygmomanometer and stethoscope

(Figure 5). The sphygmomanometer had a maximum range of $300 \mathrm{mmHg}$ and was accurate to $+3 \mathrm{mmHg}$.

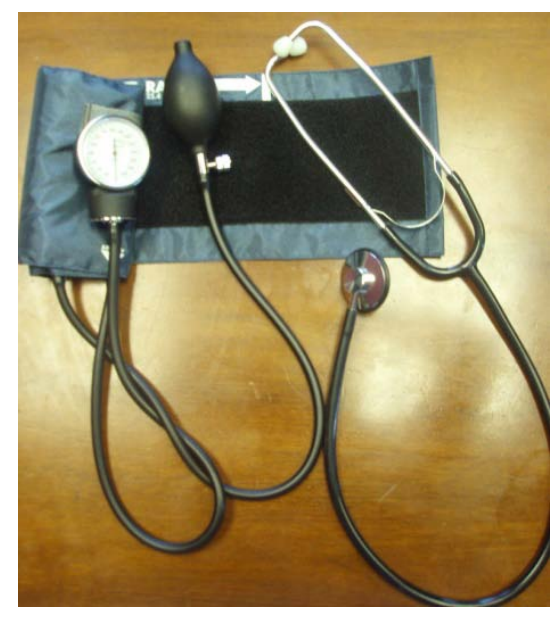

FIGURE 5 -- Moore Medical 775-660 sphygmomanometer and stethoscope 


\subsection{Pulse Oximeter}

At the time of UBM examination, oxygen saturation measurements were acquired simultaneously. The saturation of peripheral oxygen $\left(\mathrm{S}_{\mathrm{p}} \mathrm{O}_{2}\right)$ was measured in the second, third and fourth digital arteries of both the native and transplant hands for each subject with a Nonin Onyx 9500 Fingertip Pulse Oximeter (Plymouth, MN). The Nonin Onyx 9500 pulse oximeter has an oxygen saturation declared accuracy range of $70-100 \% \mathrm{SpO}_{2}$ \pm 2 digits and a range of $0-100 \% \mathrm{SpO}_{2}$.

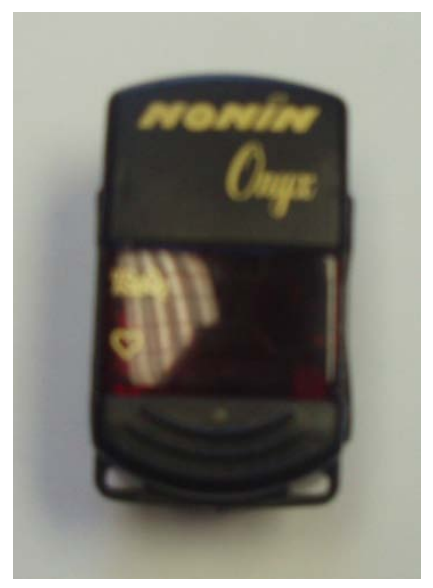

FIGURE 6 -- Nonin Onyx 9500 pulse oximeter

\subsection{Data processing}

Processing of data from the UBM as well as statistical analysis was performed on a Lenovo Y430 laptop (Lenovo, Morrisville, NC). From the manufacturer of the UBM machine, the Vevo 2100 1.1.0 (VisualSonics, Toronto, Canada) software was used to make measurements and collect still images from the UBM data. Microsoft Excel 2007 was used for tabulation of data and calculations. Statistical analyses were performed using Minitab 15 software (Minitab, State College, PA). 


\section{PROCEDURE}

Please note section 4.1 includes information about the transplantation procedures and details for each patient. Sections 4.1 and 4.2 were not performed by the author of this paper, and are included in this presentation for a more clear understanding of the research to the reader.

\section{$\underline{4.1 \text { Hand transplantation patient profiles }}$}

Deceased donors had to meet several inclusion criteria before being used for the composite tissue allotransplant (CTA). Donors had to meet the standard criteria for determination of brain death and donation after cardiac death was not considered. An ABO blood type group compatibility and a positive/negative blood type cross-match was required. Based on the preferences of the recipient and the judgment of the transplant team, matching for sex, skin tone, race, and age were sought. Donors were not included if they had active infection (HIV, hepatitis B and C, viral encephalitis), malignancy, current intravenous drug use, inherited or metabolic neuropathy, and rheumatoid or osteoarthritis. Detailed evaluations of the limbs of potential donors were performed, including range of motion in all joints, evidence of trauma/arthritis, and X-rays.

For limb procurement, a circumferential incision was made around the arm with identification of the underlying veins and nerves. A longitudinal incision was made along the medial arm over the brachial vessels to enable cannulation for cold perfusion of the limb. The limb was cooled externally by topical application of sterile saline slush and internally by perfusion of the brachial artery with University of Wisconsin solution (ViaSpan, Dupont Merck Pharmaceuticals, Wilmington, DE) at $4^{\circ} \mathrm{C}$. The resected arm 
was then wrapped in moist sponges and placed in a sterile container for transportation. For the transplantation procedure, the sequence of tissue repair was in the following order: bony fixation, arterial revascularization, vein repair, tendon repair, and nerve repair.

Brief patient histories including vascular repair descriptions, immunosuppression regimens, and adverse events are provided below for each subject investigated in this report.

Patient 1

Patient 1 received a transplantation of the left hand and forearm in January 1999. Vascular repair was carried out by and end-to-end anastomosis of the donor radial artery to the recipient radial artery, and donor ulnar artery to the recipient ulnar artery. The patient received the standard immunosuppression regimen of triple drug therapy. Specifically, patient 1 was treated with a combination of tacrolimus, mycophenolate mofetil (MMF), and prednisone. To reduce side effects from the drug therapy, patient 1 was weaned-off of the steroid (prednisone) 8 years after transplant.

Patient 1 had three episodes of acute rejection in the first year after transplantation, which were easily controlled with short courses of additional medication, generally steroids. The patient developed a viral infection common to transplant patients, cytomegalovirus (CMV), at three months after transplant that responded to medication. For patient 1, motor function improved from the end of the first year to six years and has remained relatively stable. The patient continues to experience sensory changes, even at 11 years post-transplant. 


\section{Patient 2}

Patient 2 received a transplantation of the right hand and forearm in November 2006. Vascular repair was carried out by an end-to-end anastomosis of the donor radial and ulnar arteries to the recipient radial and ulnar arteries. There was a vein graft used from the recipient on the donor ulnar artery because the ulnar vessel was not long enough. The same may also apply to the radial artery. Patient 2 received an immunosuppression induction treatment (Campath $1 \mathrm{H}$ ) that was designed to need only two types of immunosuppression. The patient was never started on steroids, but was maintained on tacrolimus and MMF. Patient 2 was the first hand transplant patient to have successfully avoided prednisone maintenance. However, patient 2 was suspected to have marginal zone lymphoma, since an unusual B-cell clone and a monoclonal T-cell clone was identified in his blood at 23 months post- transplant.

Patient 2 had three significant rejection episodes in the two years following his transplant. The first episode was a period of combine rejection and infection (CMV) at two months post transplant. The third episode of rejection was in response to decreasing immunosuppression, and was resolved once immunosuppression was restored. Patient 3

Patient 3 received a transplantation of the right hand and forearm in November 2008. Vascular repair was carried out by end-to-side anastomoses of the donor radial and ulnar arteries to the recipient's brachial artery, just proximal to the antecubital fossa. Patient 3 also received the Campath $1 \mathrm{H}$ immunosuppression and was maintained on tacrolimus and MMF, before adding prednisone to his immunosuppression a few months 
after transplantation. The patient was started on prednisone after the amputation of the graft in the fourth Louisville CTA transplant recipient.

\section{Patient 4}

Patient 4 received transplantation of the left hand and forearm in February 2001. The patient received a similar immunosuppression regimen as the first patient, with the triple drug therapy of tacrolimus, MMF, and prednisone as well as Simulect as an induction agent. Simulect is an antibody to the IL-2R. However, this patient developed high blood sugars that were difficult to control due to the high levels of tacrolimus and the multiple steroid bolus regimens used to treat rejection in the weeks following transplantation. To improve the patient's blood sugar levels, MMF was discontinued and switched to another type of immunosuppressant, rapamycin. Late in the post-transplant course, this patient was successfully weaned-off the steroids and has been maintained on tacrolimus and rapamycin.

It may be important to note that part of the patient's native arm was also injured in the explosion that caused the loss of the left hand. Patient 4 had some scaring on the native arm on the ulnar side. In addition, at the time of the UBM examination, the patient had a rash on the transplant palm and wrist. The clinicians indicated that this could be a sign of rejection or it could be a dermatological reaction. The rash was only on the transplanted hand and the clinicians claim that the transplant hand is more susceptible to dermatologic reactions. In addition, patient 4 used both hands in manual labor related to the patient's business and both hands had several calluses. These calluses appeared to impact the UBM visualization of the blood vessels subcutaneously by increasing the reflection of the acoustic beams through the tissue. 
This patient had five episodes of acute rejection in the first year that responded to short courses of increased medication. In the fifth and seventh year post-transplant, the patient had another rejection episode related to non-compliance with medication. This patient also developed osteonecrosis of both hips (one side in year 2 and one side in year 6 after transplantation), which required total hip replacement on both sides. This was directly related to the immunosuppressive regimen (prednisone and/or tacrolimus). The hand transplant team addressed this issue by implementing a steroid sparing induction regimen using Campath $1 \mathrm{H}$ in the subsequent patients, which has been routinely used in kidney transplantation.

\subsection{Ultrasound for obtaining digital brachial indices}

The systolic pressures of the brachial artery at the upper arm, the radial artery at the wrist, the ulnar artery at the wrist, and each digital artery around the proximal phalanx were determined at least once a year using a Flo-Lab 2100-SX (Parks Medical Electronics, Aloha, OR). In addition, the artery with the greatest pressure at the wrist (either the ulnar or radial) was used to determine the systolic pressure in the forearm.

A certified ultrasound technician performed the exams. Ultrasonic gel was used on the skin of the patient where the ultrasound was obtained. The cuffs were placed on both arms and a Doppler pencil probe operating at a continuous wave frequency of 8 $\mathrm{MHz}$ was used to isolate the artery of choice (except in the digital arteries). The pressure cuffs were inflated until the pressure waveform became a flat line. Next, the cuff pressure was decreased until the waveform reappeared. The technician used manual controls to determine when the pressure waveform first reappeared by moving a cursor to 
this location. This pressure value was then recorded. For the digital arteries, a much smaller cuff was used in combination with the PPG sensor, in place of the Doppler pencil probe. The brachial indices were calculated as the ratio of the systolic pressure at the specified artery over the systolic pressure of the brachial artery of the native arm. Thus, the brachial indices are normalized to the native arm.

\subsection{Ultrasound Biomicroscopy}

Ultrasonic gel was put on the patients' skin where the ultrasound was to be acquired. Each patient's arm was examined by UBM using a transducer. The frequency of the transducer was chosen based upon the depth of the artery being examined. When examining radial and ulnar arteries, a 30 or $40 \mathrm{MHz}$ transducer was generally chosen to obtain an image. If the artery was deeper, a lower frequency transducer was used. If the artery was more superficial, a higher frequency transducer was chosen to maximize resolution while balancing the limitations of penetration depth. The following arteries were examined in each patient:

TABLE II

ARTERIES EXAMINED

\begin{tabular}{|l|l|}
\hline \multicolumn{1}{|c|}{ Arm } & \multicolumn{1}{|c|}{ Artery } \\
\hline \multirow{4}{*}{ Transplant } & Brachial \\
\cline { 2 - 2 } & Ulnar \\
\cline { 2 - 2 } & Radial \\
\cline { 2 - 2 } & Palmar arch \\
\cline { 2 - 2 } & Digit 1 \\
\cline { 2 - 2 } & Digit 2 \\
\cline { 2 - 2 } & Digit 3 \\
\cline { 2 - 2 } & Digit 4 \\
\cline { 2 - 2 } & Digit 5 \\
\hline
\end{tabular}




\begin{tabular}{|l|l|}
\hline \multirow{5}{*}{ Native } & Brachial \\
\cline { 2 - 2 } & Ulnar \\
\cline { 2 - 2 } & Radial \\
\cline { 2 - 2 } & Palmar arch \\
\cline { 2 - 2 } & Digit 1 \\
\hline & Digit 2 \\
\hline & Digit 3 \\
\hline & Digit 4 \\
\hline & Digit 5 \\
\hline
\end{tabular}

Images of each arterial region of interest were acquired with a linear array transducer connected to Vevo 2100 ultrasound biomicroscopy (VisualSonics, Toronto, Canada). The resolution, frame rate and maximum tissue penetration of each transducer may be found in the previous Instrumentation and Equipment section. A cine loop, for a minimum of 4 seconds and maximum of 5 seconds, was acquired from each artery. All images were analyzed using the image analysis software, Vevo 2100 1.1.0, provided by Vevo 2100. Figure 7 shows an image taken from the cine loop of the radial artery of a transplanted arm. 


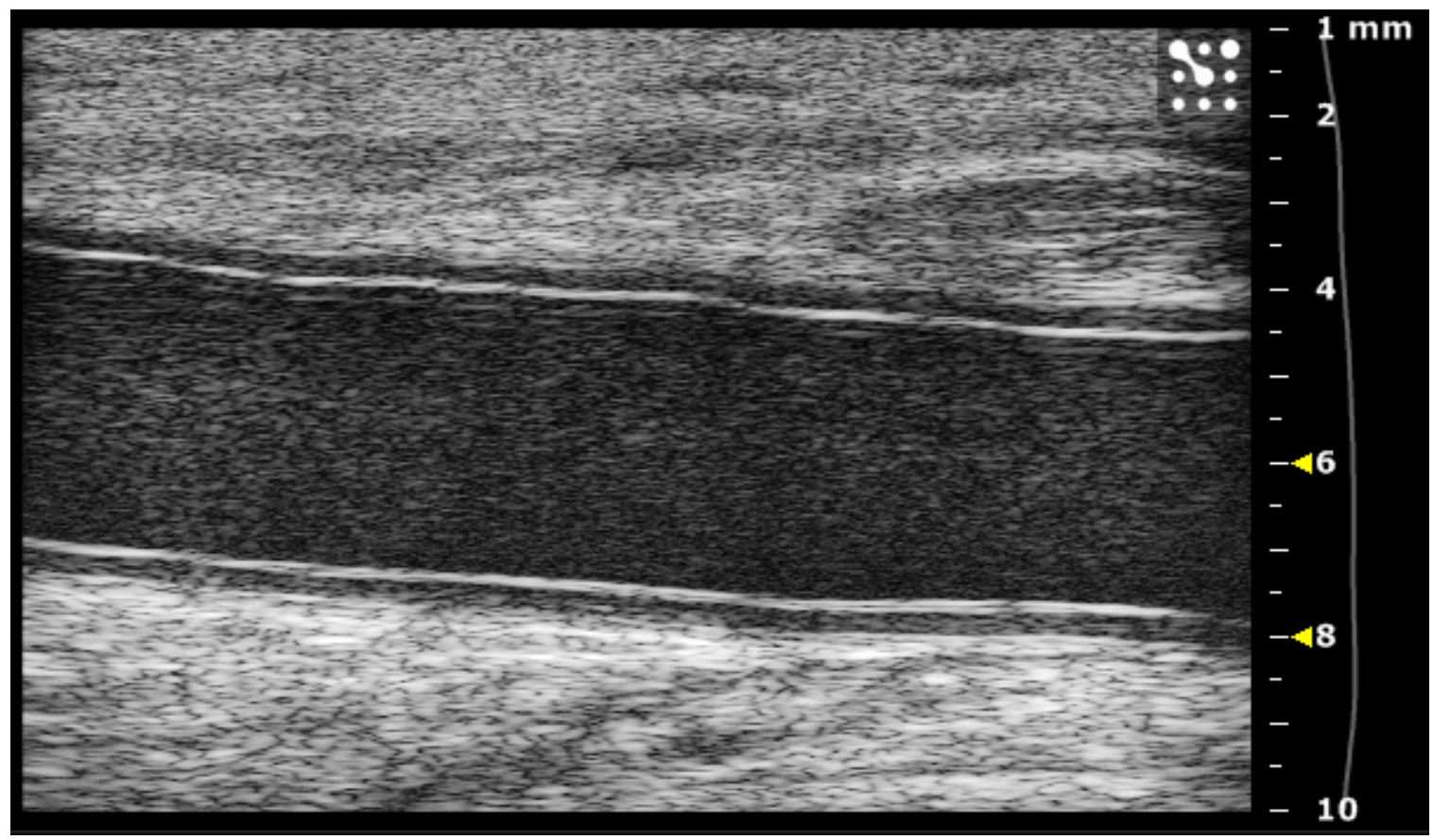

FIGURE 7 - Ultrasound image acquired by the UBM for a radial artery from a transplanted arm.

Subjects were examined sitting down with the arm being examined resting on a table. The brachial artery was examined proximal to the antecubital fossa. The ulnar and radial arteries were measured between 1 and $2 \mathrm{~cm}$ proximal to the skinfold separating the palma manus (palmar region of the hand) from regio antebrachii anterior (anterior region of the forearm) at the position of the thickest part of the far wall (visually judged). The cine loop was examined in a longitudinal projection. Data analyses were completed after patient examination using the Vevo 2100 software loaded on the Lenovo Y430 laptop computer.

\section{$\underline{4.4 \text { Arterial Measurements }}$}


Three measurements of the intima thickness (IT), intima-media thickness (IMT), and lumen diameter (LD) were performed during systole, that is, when the artery exhibited its largest diameter. IT was defined as the distance from the leading edge of the lumen-intima interface to the intima-media interface of the far wall. IMT was defined as the distance from the lumen-intima interface of the far wall to the leading edge of the media-adventitia interface. Lumen diameter was defined as the distance between the leading edges of the intima-lumen interface of the near wall and the lumen-intima interface of the far wall. The media thickness (MT) was determined by subtracting the IT from the IMT. These methods have been previously described by Wendelhag et al. (1991) and implemented in other vascular ultrasound studies as well (Johansson, 2010; Razuvaev, 2008; Osika, 2006). Figure 8 illustrates the definition of lumen, intima, and media from a UBM image of a transplanted radial artery.

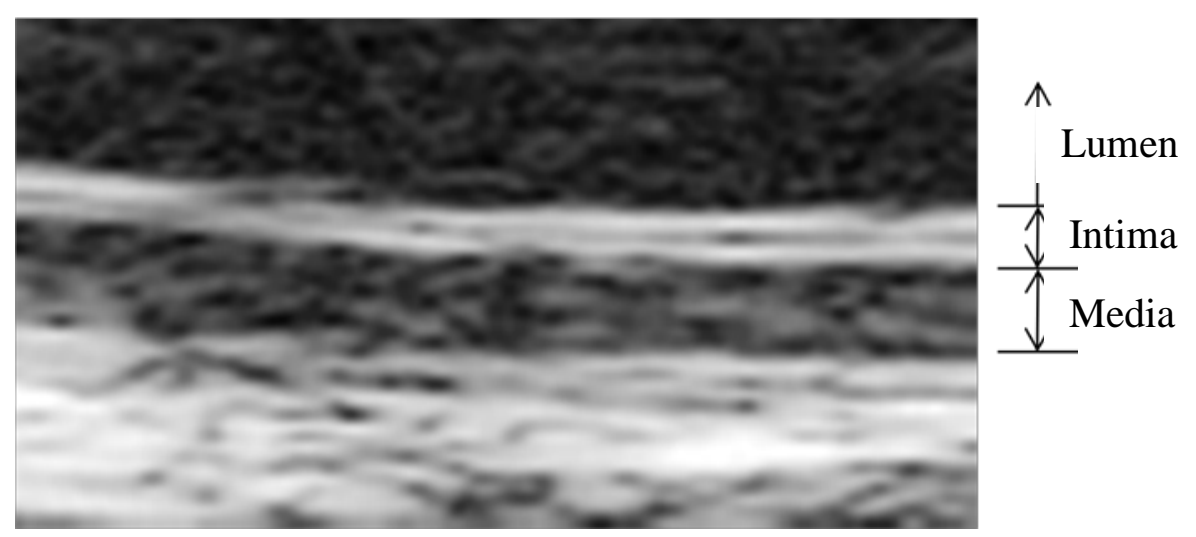

FIGURE 8 - Image defining the lumen, intima, and media layers extracted from an UBM image.

The arterial diameters varied between subjects and region of investigation. Some patients may have larger intima thicknesses than others, but they might also have larger lumen diameters. If both their lumen and intima thicknesses were larger, the level of intima in one patient may not be as severe in another patient with a smaller lumen. Thus, 
three ratios were calculated to create normalized variables for comparison across the subjects: IT/LD, IMT/LD, and MT/LD. Normalizing the measurements addresses the variability between different patients. All of these measurements were compared to the brachial indices as well, since brachial indices are the most commonly used method to assess arteriopathy in these patients.

\section{$\underline{4.5 \text { Hoop stress calculation }}$}

In order to better understand the mechanical factors involved with the intimal hyperplasia of hand transplant patients, hoop stress was calculated for each vessel. Hoop stress is the amount of stress which occurs in the circumferential direction in a thinwalled vessel. The hoop stress is defined by the following equation:

In this study, calculations of the hoop stress of the arterial walls were performed using data from the pressure measurements obtained with the Flo-Lab 2100 and arterial dimensions acquired by the UBM. Specifically, the variables in the equation are: $\mathrm{p}=$ pressure in the artery $(\mathrm{mmHg}), \mathrm{D}=$ lumen diameter $(\mathrm{mm})$, and $\mathrm{t}=$ =wall thickness of the artery. The pressure values used were obtained from the ultrasound to obtain the brachial indices. The lumen diameter values used were the LD measurements from the UBM examination. Lastly, the wall thickness was identified as the intima-media thickness (IMT), which was also obtained from the UBM examination.

\section{$\underline{4.6 \text { Blood pressure measurement }}$}


A registered nurse took blood pressure measurements of each patient before and after the UBM examination using a professional aneroid sphygmomantometer and stethoscope. Patients sat upright in a chair, resting their arms on a table. Blood pressure measurements were read aloud and noted.

\subsection{Oxygen saturation measurement}

Oxygen saturation of the second, third, and fourth digits on each hand for each patient was measured using a pulse oximeter before the UBM examination. As with the blood pressure measurements, patients sat in a chair, resting their arms on a table. Oxygen saturation percentages were read aloud and noted.

\section{$\underline{4.8 \text { Statistical Analysis }}$}

Because of the small samples size available, demonstrating normal distribution of the data is difficult. Thus, the nonparametric equivalent of a one-way analysis of variance (ANOVA), the Kruskall-Wallis test, was used to determine statistical significance, defined as $\mathrm{p}<0.05$. Data was copied from Microsoft Excel 2007 into Minitab 15. Within Minitab, averages of parameters from the transplanted and native hands were calculated. Then, the Kruskall-Wallis test was performed in Minitab to determine significance. Comparisons were made using the Pearson correlation coefficient. 


\section{RESULTS AND DISCUSSION OF RESULTS}

\section{$\underline{5.1 \text { Brachial index measurements }}$}

A goal of this project was to compare the relationships that existed between brachial indices and transplanted arteries versus native arteries. Defined as the pressure ratio from the indicated artery to the brachial artery, the use of the brachial index to assess perfusion in hand transplant patients is questioned. Clinical practice regards brachial indices of 0.7 or less as an indication of inadequate blood flow to the indicated artery. The first patient examined, who has had their graft for 11 years, was the only patient to have at least one brachial index below the 0.7 threshold. Overall, the brachial indices of this patient's transplanted arm were much lower than his native arm (Table III). For example, the average difference between brachial indices of transplanted artery versus the native artery on the contralateral arm was 0.11 . This large difference between arms suggests that the patient's transplanted arm is not being perfused as well as their native arm. However, this data does not provide a clear indication of whether the patient has a significant level of arteriopathy that warrants a change in immunosuppression in order to protect the graft from ischemia. In addition, this patient's digital brachial indices ranged from 0.69 (digit 5) to 0.81 (digit 3), with an average value of 0.75 . Even though some of the brachial indices fell below the prescribed threshold value, the clinicians decided not to change his immunosuppression.

Patient 2, at 3 years post-transplant, had just slightly lower brachial indices in his transplanted arm, with an average difference of 0.06 . His lowest index was with digit 2 being 0.77 . Patients 3 and 4 had much higher brachial indices on both the transplanted 
and native arms than the first two patients. Patient 3, at 15 months post-transplant, had a lowest brachial index of 0.89 in the transplanted digit 5 . Patient 4 , at 9 years posttransplant, had a lowest brachial index of 1.10 in the transplanted digit 1 . Thus, from a clinical viewpoint, patients 3 and 4 showed no sign of compromised blood flow, which could be caused by an obstructive phenomenon such as intimal hyperplasia. However, though patients 3 and 4 had the highest brachial indices, they exhibited the largest differences between arms.

A Kruskal-Wallis, nonparametric statistical analysis, was performed on the data to determine if the condition of being a transplanted artery significantly affected the brachial index value. An analysis was performed using each patient separately and another analysis was performed using the data from all four patients together. From the data analyzing each patient separately, only patients 3 and 4 were found to have significantly ( $\mathrm{p}=0.007$ and $\mathrm{p}=0.022$, respectively) lower brachial indices in their transplanted hand and forearm. This was a bit surprising, considering these patients were also found to have the highest brachial indices. Although patient 1 was close to statistical significance, the pvalue of 0.064 did not meet our statistical significance cut-off criteria of $p=0.05$. From the statistical analysis using the data from all four patients together, the transplanted arm was found to have significantly $(\mathrm{p}=0.037)$ lower brachial indices than in the contralateral arm.

From a clinical perspective, these results suggest that the arteries of the transplanted hand do not have the same level vascular perfusion as the native arm. Currently, clinicians used brachial indices to eliminate the suspicion of any obstructive phenomenon which could compromise blood flow, such as intimal hyperplasia. 
However, these results do not provide useful information regarding the presence and level of arteriopathy found in those arteries. The author has been unable to find studies that included brachial index measurement besides the very limited data in the papers that establish the clinical threshold of 0.7 . 
TABLE III

BRACHIAL INDEX RESULTS

\begin{tabular}{|c|c|c|c|c|c|c|c|c|}
\hline \multirow[b]{2}{*}{ Subject } & \multicolumn{8}{|c|}{ Transplant Brachial Index } \\
\hline & Ulnar & Radial & Palmar Arch & Digit 1 & Digit 2 & Digit 3 & Digit 4 & Digit 5 \\
\hline 1 & 0.99 & 0.97 & & 0.79 & 0.76 & 0.81 & 0.72 & 0.69 \\
\hline 2 & 0.93 & 0.88 & & 0.80 & 0.77 & 0.78 & 0.81 & 0.81 \\
\hline 3 & 1.05 & 1.05 & & 1.02 & 0.96 & 1.09 & 0.93 & 0.89 \\
\hline 4 & 1.25 & 1.23 & & 1.10 & 1.18 & 1.27 & 1.19 & 1.22 \\
\hline Average & $1.055 \pm 0.139$ & $1.033 \pm 0.149$ & & $0.928 \pm 0.156$ & $0.918 \pm 0.198$ & $0.988 \pm 0.234$ & $0.913 \pm 0.204$ & $0.903 \pm 0.227$ \\
\hline
\end{tabular}

\begin{tabular}{|c|c|c|c|c|c|c|c|c|}
\hline \multirow[b]{2}{*}{ Subject } & \multicolumn{8}{|c|}{ Native Brachial Index } \\
\hline & Ulnar & Radial & Palmar Arch & Digit 1 & Digit 2 & Digit 3 & Digit 4 & Digit 5 \\
\hline 1 & 1.10 & 1.01 & & 0.80 & 0.92 & 0.97 & 0.89 & 0.92 \\
\hline 2 & 0.99 & 1.14 & & 0.80 & 0.75 & 0.80 & 0.88 & 0.91 \\
\hline 3 & 1.11 & 1.15 & & 1.11 & 1.19 & 1.02 & 1.50 & 1.16 \\
\hline 4 & 1.39 & 1.48 & & 1.22 & 1.50 & 1.30 & 1.46 & 1.24 \\
\hline Average & $1.148 \pm 0.171$ & $1.195 \pm 0.2$ & & $0.983 \pm 0.215$ & $1.09 \pm 0.328$ & $1.023 \pm 0.208$ & $1.183 \pm 0.344$ & $1.058 \pm 0.168$ \\
\hline
\end{tabular}

Note: The Palmar Arch column was intentionally left blank. 


\subsection{Ultrasound Biomicroscopy measurements}

For the first time in clinical history, a clinical tool has become available to now enable clinicians to monitor the thickening of the arterial wall over time. The UBM has provided a way to non-invasively and accurately measure the same area over time with real-time results. This will give the clinician valuable information regarding the progression of chronic rejection. Unlike acute rejection, chronic rejection does not come in episodes. It is a single, progressive event that can take several months (as with the recipient who lost their graft at the Louisville CTA Program) or several years to manifest into a threat to the viability of the transplant. Using the protocol described here, effects of particular treatments and conditions (i.e. changing immunosuppression treatments, mechanical factors, etc.) may be investigated to understand their effects on chronic rejection.

In total, 255 cine loops were collected with the UBM in this study. Each cine loop includes 100 image frames. Only a portion of the cine loops were used for analysis. This was for many reasons: the image would blur once it had started recording the cine loop, the operator trying to obtain a better image, or the clinicians finding anomalies unrelated to this project that they wanted to record. A total of 59 images were analyzed. Nine measurements were taken in each image: 3 intima, 3 intima-media, and 3 lumen diameter. Thus, there were a total of 531 raw measurements of the UBM data.

\subsubsection{Intima Thickness}

One hypothesis of the project was that the intima thicknesses are significantly larger in the transplanted hand than in the native hand. In each patient the radial and 
ulnar arteries, palmar arch, and each of the five digital arteries were examined using the UBM. However, in patients 1 and 3, not every artery was examined due, for the most part, to inherent limitations of the ultrasound system. In particular, with ultrasound transducers, there is a compromise between frequency (and, therefore, resolution) and acoustic depth of penetration, where the depth of penetration is inversely proportional to the operating frequency of the ultrasound transducer as seen in the following equation:

Where $\mathrm{Z}_{\max }$ is penetration depth, $\mathrm{v}_{\max }$ is the maximum velocity, $\mathrm{c}$ is the speed of sound, and $f_{\mathrm{o}}$ is the operating frequency. Assuming the maximum velocity is constant, it is seen that as the frequency is increased, the depth of penetration or range of the acoustic beam is decreased.

Since some patients had much larger arms than others, the depth of penetration increased for the ultrasound to travel in order to image the arteries of interest. Since some patients had swelling, inflammation or scarring of the hand, the depth of penetration for imaging the arteries of interest dramatically increased, which directly influenced the ability to acquire an adequate image. In addition, if the patient had swelling or inflammation of the hand, this also increased the depth of penetration dramatically. Another potential cause for the inability to acquire data in some arterial vessels was the UBM operator having difficulty locating the digital arteries. The arterial anatomy of the hand is complex and highly variable from person-to-person. Maneuvering the transducer around the digits also introduced issues, especially since ultrasound has difficulty penetrating bone. Much of the volume of the digits is composed 
of bone. The physical size of the transducer was sometimes too large to acquire a clear image of the digital arteries, since the edges of the transducer would extend over the finger.

The smallest intima thickness (Table IV) found was $0.052 \mathrm{~mm}$ in the native second digital artery of patient 1 . The largest intima thickness found was $0.177 \mathrm{~mm}$ in the transplanted radial artery of patient 2. The intima in almost all arteries was larger in the transplanted hand compared to the native hand. As with the brachial index, a Kruskal-Wallis test was used to determine if the intima of the arteries in the transplanted hand were significantly larger than the intima of the arteries in the native hand. From the statistical analysis using the combined data from all four of the patients, the transplanted hand was found to have significantly $(\mathrm{p}=0.001)$ larger intima thicknesses than the native hand. Thus, the first hypothesis is accepted: the intima thicknesses are significantly higher in the transplanted hand, using the patient as his own control. 
TABLE IV

INTIMA THICKNESS RESULTS

\begin{tabular}{|c|c|c|c|c|c|c|c|c|}
\hline & \multicolumn{9}{|c|}{ Transplant IT (mm) } & Digit 3 & Digit 4 & Digit 5 \\
\hline Subject & UInar & Radial & Palmar Arch & Digit 1 & Digit 2 & & 0.074 & 0.100 \\
\hline 1 & 0.152 & 0.119 & & & & 0.095 \\
\hline 2 & 0.122 & 0.177 & 0.094 & 0.060 & 0.090 & 0.103 & 0.096 & 0.105 \\
\hline 3 & 0.121 & 0.121 & 0.108 & & 0.090 & 0.074 & 0.079 & 0.084 \\
\hline 4 & 0.14 & 0.145 & 0.12 & 0.066 & 0.102 & 0.091 & 0.102 & 0.132 \\
\hline Average & $0.134 \pm 0.015$ & $0.141 \pm 0.027$ & $0.107 \pm 0.013$ & $0.063 \pm 0.004$ & $0.094 \pm 0.007$ & $0.086 \pm 0.014$ & $0.094 \pm 0.01$ & $0.104 \pm 0.021$ \\
\hline
\end{tabular}

\begin{tabular}{|c|c|c|c|c|c|c|c|c|}
\hline & \multicolumn{9}{|c|}{ Native IT (mm) } \\
\hline Subject & Ulnar & Radial & Palmar Arch & Digit 1 & Digit 2 & Digit 3 & Digit 4 & Digit 5 \\
\hline 1 & 0.090 & 0.090 & & 0.084 & 0.052 & 0.102 & 0.079 & 0.085 \\
\hline 2 & 0.084 & 0.108 & 0.099 & 0.072 & 0.054 & 0.060 & 0.066 & 0.090 \\
\hline 3 & 0.109 & 0.117 & 0.090 & 0.064 & 0.070 & 0.063 & 0.077 & 0.070 \\
\hline 4 & 0.100 & 0.120 & 0.120 & 0.054 & 0.084 & 0.066 & 0.067 & 0.060 \\
\hline Average & $0.096 \pm 0.011$ & $0.109 \pm 0.013$ & $0.103 \pm 0.015$ & $0.069 \pm 0.013$ & $0.065 \pm 0.015$ & $0.073 \pm 0.02$ & $0.072 \pm 0.007$ & $0.076 \pm 0.014$ \\
\hline
\end{tabular}


Small levels of intimal hyperplasia have been found to be normal, which progresses with age (Subbotin, 2007; Osika, 2007). The defining feature of chronic rejection has been defined as the progressive thickening of the arterial walls within the affected organ due to intimal hyperplasia (Geraghty, 1996; Merville, 2005; Hayry, 1991; Ross, 1993). The results of this investigation indicated that the intima of the arteries in the transplanted hand were larger than in the native hand by an average of $0.022 \mathrm{~mm}$. Nonetheless, no UBM image from the patients involved in this study suggested that the thickening was so severe as to threaten the vascular supply to the hand.

This is the first known study that compares IH between native and transplanted hands. The increased IH likely indicates early signs of chronic rejection in each of the patient. The levels of IH found do not seem to threaten the viability of any of the transplants in the near future. However, it is crucial that the clinicians monitor the progression of IH in these patients. It is not clear what level of IH would warrant increases in immunosuppression, but the UBM provides a real-time image of the arterial cross-section. From there, the physician can make a clinical decision as to whether or not extra measures should be taken. In addition, it is vital that the patients adhere to their immunosuppression. If they did not take their immunosuppression, the IH would grow much more rapidly.

\subsubsection{Intima-media and media thicknesses}

Using a Kruskal-Wallis test, it was found that the IMT of the transplanted hand was not significantly larger than in the native hand. The average IMT (Table V) for the transplanted hand was $0.204 \mathrm{~mm}$, while the native hand had an average IMT of 0.187 
$\mathrm{mm}$. The effect of chronic rejection is seen in the intima layers of arteries (Geraghty, 1996; Merville, 2005; Hayry, 1991; Ross, 1993). Since the media makes up the bulk of the arterial wall (Shier, 2006), it is logical that the IMT is not significant because the media layer is dominating these measurement.

The media thickness (MT) was calculated by subtracting the IT from the IMT in each artery. Like the IMT, the MT (Table VI) of the arteries in the transplanted hand was not significantly different than in the native hand. The average MT of the native hand $(0.105 \mathrm{~mm})$ was slightly larger than in the transplanted hand $(0.101 \mathrm{~mm})$. Again, since the hallmark characteristic of chronic rejection is increased intimal thickness, it is logical that the media thicknesses not significantly different between the arms. Chronic rejection does not affect the medial layer. 
TABLE V

INTIMA-MEDIA THICKNESS RESULTS

\begin{tabular}{|c|c|c|c|c|c|c|c|c|}
\hline & \multicolumn{9}{|c|}{ Transplant IMT (mm) } \\
\hline Subject & Ulnar & Radial & Palmar Arch & Digit 1 & Digit 2 & Digit 3 & Digit 4 & Digit 5 \\
\hline 1 & 0.284 & 0.272 & & & 0.303 & 0.235 \\
\hline 2 & 0.252 & 0.207 & 0.127 & 0.114 & 0.144 & 0.146 & 0.138 & 0.111 \\
\hline 3 & 0.198 & 0.194 & 0.225 & & 0.144 & 0.134 & 0.137 & 0.105 \\
\hline 4 & 0.509 & 0.360 & 0.259 & 0.138 & 0.192 & 0.192 & 0.228 \\
\hline Average & $0.311 \pm 0.137$ & $0.258 \pm 0.076$ & $0.204 \pm 0.069$ & $0.126 \pm 0.017$ & $0.16 \pm 0.028$ & $0.194 \pm 0.077$ & $0.185 \pm 0.054$ & $0.147 \pm 0.049$ \\
\hline
\end{tabular}

\begin{tabular}{|c|c|c|c|c|c|c|c|c|}
\hline \multirow[b]{2}{*}{ Subject } & \multicolumn{8}{|c|}{ Native IMT (mm) } \\
\hline & Ulnar & Radial & Palmar Arch & Digit 1 & Digit 2 & Digit 3 & Digit 4 & Digit 5 \\
\hline 1 & 0.265 & 0.270 & & 0.196 & 0.149 & 0.144 & 0.177 & 0.134 \\
\hline 2 & 0.330 & 0.270 & 0.180 & 0.108 & 0.120 & 0.132 & 0.126 & 0.144 \\
\hline 3 & 0.189 & 0.234 & 0.207 & 0.084 & 0.133 & 0.140 & 0.112 & 0.112 \\
\hline 4 & 0.240 & 0.339 & 0.250 & 0.162 & 0.246 & 0.216 & 0.181 & 0.210 \\
\hline Average & $0.256 \pm 0.059$ & $0.278 \pm 0.044$ & $0.212 \pm 0.035$ & $0.138 \pm 0.051$ & $0.162 \pm 0.057$ & $0.158 \pm 0.039$ & $0.149 \pm 0.035$ & $0.15 \pm 0.042$ \\
\hline
\end{tabular}


TABLE VI

MEDIA THICKNESS RESULTS

\begin{tabular}{|c|c|c|c|c|c|c|c|c|}
\hline & \multicolumn{8}{|c|}{ Transplant MT (mm) } \\
\hline Subject & Ulnar & Radial & Palmar Arch & Digit 1 & Digit 2 & Digit 3 & Digit 4 & Digit 5 \\
\hline 1 & 0.132 & 0.154 & & & & 0.229 & 0.135 & 0.066 \\
\hline 2 & 0.130 & 0.090 & 0.033 & 0.054 & 0.053 & 0.043 & 0.041 & 0.006 \\
\hline 3 & 0.077 & 0.073 & 0.117 & & 0.054 & 0.059 & 0.058 & 0.021 \\
\hline 4 & 0.369 & 0.215 & 0.139 & 0.072 & 0.090 & 0.101 & 0.126 & 0.078 \\
\hline Average & $0.177 \pm 0.131$ & $0.133 \pm 0.065$ & $0.096 \pm 0.056$ & $0.063 \pm 0.013$ & $0.066 \pm 0.021$ & $0.108 \pm 0.084$ & $0.09 \pm 0.047$ & $0.043 \pm 0.035$ \\
\hline
\end{tabular}

\begin{tabular}{|c|c|c|c|c|c|c|c|c|}
\hline \multirow[b]{2}{*}{ Subject } & \multicolumn{8}{|c|}{ Native MT (mm) } \\
\hline & Ulnar & Radial & Palmar Arch & Digit 1 & Digit 2 & Digit 3 & Digit 4 & Digit 5 \\
\hline 1 & 0.176 & 0.180 & & 0.112 & 0.097 & 0.041 & 0.098 & 0.050 \\
\hline 2 & 0.246 & 0.162 & 0.081 & 0.036 & 0.066 & 0.072 & 0.060 & 0.054 \\
\hline 3 & 0.080 & 0.117 & 0.117 & 0.020 & 0.063 & 0.077 & 0.035 & 0.043 \\
\hline 4 & 0.140 & 0.219 & 0.130 & 0.108 & 0.162 & 0.150 & 0.114 & 0.150 \\
\hline Average & $0.161 \pm 0.069$ & $0.17 \pm 0.042$ & $0.109 \pm 0.025$ & $0.069 \pm 0.048$ & $0.097 \pm 0.046$ & $0.085 \pm 0.046$ & $0.077 \pm 0.036$ & $0.074 \pm 0.051$ \\
\hline
\end{tabular}




\subsubsection{Lumen diameter}

The UBM examinations showed that all of the arteries examined were patent, allowing adequate blood flow to the hand. Results of the Kruskal-Wallis statistical analysis found that lumen diameter was near statistical significance with $\mathrm{p}=0.066$. On average, lumen diameters were smaller in the transplanted hand of all four patients. The largest lumen diameter difference was seen in the radial artery, where the lumen diameter was $0.956 \mathrm{~mm}$ smaller in transplanted hand on average compared to the native hand across all four patients.

Lumen diameter measurements provide valuable information about the crosssectional area through which the blood passes. If the lumen diameter is very small, less blood will be able to be delivered. In the Louisville CTA hand transplant recipient who lost their graft, histological analysis of several arteries, including radial and ulnar arteries, revealed diminutive lumen diameters due to severe intimal hyperplasia. The area that had been part of the lumen was overcome by the rapidly growing intima. The catastrophic result was almost complete restriction of blood flow to the hand, which finally led to thrombosis and amputation of the graft. This patient was on the lowest initial dose of immunosuppression that the Louisville CTA program had used, with only two drugs and no steroids. Thus, chronic rejection manifested into a catastrophic event much more quickly than expected. If the clinicians had the UBM system used in this study, they may have been able to salvage the patient's arm by implementing alternative drug therapies to halting the IH growth. They would have known how severe the patient's IH was in realtime, without the need for histological analysis of a biopsy. 
TABLE VII

LUMEN DIAMETER RESULTS

\begin{tabular}{|c|c|c|c|c|c|c|c|c|}
\hline \multirow[b]{2}{*}{ Subject } & \multicolumn{8}{|c|}{ Transplant LD (mm) } \\
\hline & Ulnar & Radial & Palmar Arch & Digit 1 & Digit 2 & Digit 3 & Digit 4 & Digit 5 \\
\hline 1 & 1.972 & 1.432 & & & & 0.515 & 0.593 & 0.409 \\
\hline 2 & 2.341 & 1.662 & 1.385 & 0.576 & 0.671 & 0.870 & 0.907 & 0.761 \\
\hline 3 & 2.075 & 1.681 & 0.853 & & 0.943 & 0.971 & 0.780 & 0.797 \\
\hline 4 & 2.116 & 3.198 & 1.348 & 0.725 & 1.019 & 1.032 & 0.833 & 0.683 \\
\hline Average & $2.126 \pm 0.156$ & $1.993 \pm 0.811$ & $1.195 \pm 0.297$ & $0.651 \pm 0.105$ & $0.878 \pm 0.183$ & $0.847 \pm 0.231$ & $0.778 \pm 0.134$ & $0.663 \pm 0.176$ \\
\hline
\end{tabular}

\begin{tabular}{|c|c|c|c|c|c|c|c|c|}
\hline & \multicolumn{7}{|c|}{ Native LD (mm) } \\
\hline Subject & Ulnar & Radial & Palmar Arch & Digit 1 & Digit 2 & Digit 3 & Digit 4 & Digit 5 \\
\hline 1 & 1.972 & 2.066 & & 0.895 & 0.731 & 0.677 & 1.028 & 1.053 \\
\hline 2 & 1.389 & 2.847 & 1.638 & 1.079 & 1.013 & 1.347 & 0.869 & 0.946 \\
\hline 3 & 3.182 & 3.621 & 1.949 & 1.432 & 1.594 & 1.663 & 1.251 & 1.195 \\
\hline 4 & 1.427 & 3.264 & 0.918 & 0.521 & 0.977 & 0.982 & 0.826 & 0.946 \\
\hline Average & $1.993 \pm 0.837$ & $2.95 \pm 0.669$ & $1.502 \pm 0.529$ & $0.982 \pm 0.379$ & $1.079 \pm 0.366$ & $1.167 \pm 0.429$ & $0.994 \pm 0.192$ & $1.035 \pm 0.118$ \\
\hline
\end{tabular}




\subsubsection{Intima-to-lumen diameter ratios}

One goal of the project was to compare the IT-to-LD ratio of the arteries in the transplanted hand to those in the native hand. Again using the Kruskal-Wallis statistical analysis, it was found that the average IT-to-LD ratios among the four patients were significantly ( $p=0.000)$ larger in the transplanted hand $(0.105 \pm 0.042)$ compared to the native hand $(0.066 \pm 0.028)$. Thus, the second hypothesis is accepted.

This parameter is important to consider since it was normalized to each patient. The largest IT-to-LD ratio found was in the fifth digit of the transplanted hand in patient 1 (Table VIII). Interestingly, this artery was also the artery with the lowest digital brachial index of 0.69. Again, the results seen are due to the effects of chronic rejection of the transplanted grafts. Chronic rejection increases the IH, and increases the IT-to-LD ratio. Because this is a normalized value, it provides the ability to compare measurements between arms and between patients. Comparisons are more meaningful since this parameter is normalized. Of all of the parameters evaluated, the IT-to-LD ratio most directly quantifies the patency of the vessel. The ratio shows how large the intima is in relation to the lumen diameter, which allows interpretation of the severity of IH (and, thus, chronic rejection). The IT-to-LD ratio is a vital parameter to monitor in these patients in the future. 
TABLE VIII

INTIMA-TO-LUMEN DIAMETER RESULTS

\begin{tabular}{|c|c|c|c|c|c|c|c|c|}
\hline \multirow[b]{2}{*}{ Subject } & \multicolumn{8}{|c|}{ Transplant IT/LD } \\
\hline & Ulnar & Radial & Palmar Arch & Digit 1 & Digit 2 & Digit 3 & Digit 4 & Digit 5 \\
\hline 1 & 0.081 & 0.083 & & & & 0.144 & 0.168 & 0.232 \\
\hline 2 & 0.052 & 0.070 & 0.068 & 0.104 & 0.135 & 0.119 & 0.106 & 0.138 \\
\hline 3 & 0.058 & 0.072 & 0.126 & & 0.095 & 0.077 & 0.101 & 0.105 \\
\hline 4 & 0.066 & 0.045 & 0.089 & 0.091 & 0.100 & 0.088 & 0.122 & 0.193 \\
\hline Average & $0.064 \pm 0.013$ & $0.068 \pm 0.016$ & $0.094 \pm 0.03$ & $0.098 \pm 0.009$ & $0.11 \pm 0.022$ & $0.107 \pm 0.03$ & $0.124 \pm 0.03$ & $0.167 \pm 0.057$ \\
\hline
\end{tabular}

\begin{tabular}{|c|c|c|c|c|c|c|c|c|}
\hline \multirow[b]{2}{*}{ Subject } & \multicolumn{8}{|c|}{ Native IT/LD } \\
\hline & Ulnar & Radial & Palmar Arch & Digit 1 & Digit 2 & Digit 3 & Digit 4 & Digit 5 \\
\hline 1 & 0.046 & 0.043 & & 0.094 & 0.071 & 0.151 & 0.076 & 0.080 \\
\hline 2 & 0.060 & 0.038 & 0.060 & 0.067 & 0.053 & 0.044 & 0.076 & 0.095 \\
\hline 3 & 0.034 & 0.032 & 0.046 & 0.045 & 0.044 & 0.038 & 0.061 & 0.058 \\
\hline 4 & 0.070 & 0.037 & 0.131 & 0.104 & 0.086 & 0.067 & 0.081 & 0.063 \\
\hline Average & $0.053 \pm 0.016$ & $0.037 \pm 0.005$ & $0.079 \pm 0.046$ & $0.077 \pm 0.027$ & $0.064 \pm 0.019$ & $0.075 \pm 0.052$ & $0.073 \pm 0.009$ & $0.074 \pm 0.017$ \\
\hline
\end{tabular}


5.2.6 Intima-media-to-lumen diameter ratio and media-to-lumen diameter ratios

The IMT-to-lumen diameter ratio (Table IX) was found to be significantly $(p=0.035)$ larger in the transplanted hand. The average IMT-to-lumen ratio in the transplant hand was 0.2030 , while the average in the native hand was 0.1504 . These results were interesting considering that the IMT data between patients was not found to be significant. This suggested that for this normalized parameter, the effect of IH has a greater influence on the statistical significance compared to the IMT data alone. The normalized data enhances the effects of variability between the transplanted and native hands by making all measurements more accurately comparable. Thus, the effects of chronic rejection are more easily recognized in a normalized parameter.

The MT-to-lumen ratio (Table X) was not significantly different in opposing hands. The average MT-to-lumen ratio in the transplant hand was 0.0983. Similarly, the average MT-to-lumen ratio in the native hand was 0.0841. Again, since chronic rejection affects the intima, these results are logical. The normalized data still provides a more accurate comparison between hands and between patients. Yet, this parameter was not significant, as expected. This helps confirm the poorly understood notion that chronic rejection manifests as $\mathrm{IH}$, and does not have significant effects on the media layer. 
TABLE IX

INTIMA-MEDIA-TO-LUMEN DIAMETER RESULTS

\begin{tabular}{|c|c|c|c|c|c|c|c|c|}
\hline \multirow[b]{2}{*}{ Subject } & \multicolumn{8}{|c|}{ Transplant IMT/LD } \\
\hline & Ulnar & Radial & Palmar Arch & Digit 1 & Digit 2 & Digit 3 & Digit 4 & Digit 5 \\
\hline 1 & 0.152 & 0.190 & & & & 0.588 & 0.396 & 0.393 \\
\hline 2 & 0.108 & 0.124 & 0.091 & 0.198 & 0.214 & 0.168 & 0.152 & 0.145 \\
\hline 3 & 0.096 & 0.115 & 0.263 & & 0.152 & 0.138 & 0.176 & 0.131 \\
\hline 4 & 0.241 & 0.113 & 0.192 & 0.190 & 0.188 & 0.186 & 0.274 & 0.307 \\
\hline Average & $0.149 \pm 0.066$ & $0.136 \pm 0.037$ & $0.182 \pm 0.086$ & $0.194 \pm 0.006$ & $0.185 \pm 0.031$ & $0.27 \pm 0.213$ & $0.25 \pm 0.111$ & $0.244 \pm 0.127$ \\
\hline
\end{tabular}

\begin{tabular}{|c|c|c|c|c|c|c|c|c|}
\hline & \multicolumn{7}{|c|}{ Native IMT/LD } \\
\hline Subject & Ulnar & Radial & Palmar Arch & Digit 1 & Digit 2 & Digit 3 & Digit 4 & Digit 5 \\
\hline 1 & 0.135 & 0.131 & & 0.219 & 0.203 & 0.212 & 0.172 & 0.128 \\
\hline 2 & 0.237 & 0.095 & 0.110 & 0.100 & 0.118 & 0.098 & 0.145 & 0.152 \\
\hline 3 & 0.059 & 0.064 & 0.106 & 0.059 & 0.083 & 0.084 & 0.089 & 0.094 \\
\hline 4 & 0.168 & 0.104 & 0.272 & 0.311 & 0.252 & 0.220 & 0.219 & 0.222 \\
\hline Average & $0.15 \pm 0.074$ & $0.099 \pm 0.028$ & $0.163 \pm 0.095$ & $0.172 \pm 0.115$ & $0.164 \pm 0.077$ & $0.154 \pm 0.072$ & $0.156 \pm 0.054$ & $0.149 \pm 0.054$ \\
\hline
\end{tabular}


TABLE X

\section{MEDIA-TO-LUMEN DIAMETER RESULTS}

\begin{tabular}{|c|c|c|c|c|c|c|c|c|}
\hline & \multicolumn{8}{|c|}{ Transplant MT/LD } \\
\hline Subject & Ulnar & Radial & Palmar Arch & Digit 1 & Digit 2 & Digit 3 & Digit 4 & Digit 5 \\
\hline 1 & 0.071 & 0.107 & & & & 0.444 & 0.228 & 0.161 \\
\hline 2 & 0.056 & 0.054 & 0.024 & 0.094 & 0.079 & 0.049 & 0.046 & 0.008 \\
\hline 3 & 0.037 & 0.043 & 0.137 & & 0.057 & 0.061 & 0.075 & 0.026 \\
\hline 4 & 0.174 & 0.067 & 0.103 & 0.099 & 0.088 & 0.098 & 0.151 & 0.114 \\
\hline Average & $0.085 \pm 0.061$ & $0.068 \pm 0.028$ & $0.088 \pm 0.058$ & $0.097 \pm 0.004$ & $0.075 \pm 0.016$ & $0.163 \pm 0.188$ & $0.125 \pm 0.082$ & $0.077 \pm 0.073$ \\
\hline
\end{tabular}

\begin{tabular}{|c|c|c|c|c|c|c|c|c|}
\hline & \multicolumn{8}{|c|}{ Native MT/LD } \\
\hline Subject & Ulnar & Radial & Palmar Arch & Digit 1 & Digit 2 & Digit 3 & Digit 4 & Digit 5 \\
\hline 1 & 0.089 & 0.087 & & 0.125 & 0.132 & 0.061 & 0.095 & 0.047 \\
\hline 2 & 0.177 & 0.057 & 0.049 & 0.033 & 0.065 & 0.053 & 0.069 & 0.057 \\
\hline 3 & 0.025 & 0.032 & 0.060 & 0.014 & 0.039 & 0.046 & 0.028 & 0.036 \\
\hline 4 & 0.098 & 0.067 & 0.142 & 0.207 & 0.166 & 0.153 & 0.138 & 0.159 \\
\hline Average & $0.097 \pm 0.062$ & $0.061 \pm 0.023$ & $0.084 \pm 0.051$ & $0.095 \pm 0.089$ & $0.101 \pm 0.059$ & $0.078 \pm 0.05$ & $0.083 \pm 0.046$ & $0.075 \pm 0.057$ \\
\hline
\end{tabular}




\subsection{Blood pressure measurement}

The blood pressure of each patient was recorded before and after each UBM exam to check for any effects that the blood pressures may have on the other parameters and to check for significant changes in blood pressure during the examination. In addition, alterations in the blood pressure provided more insight into possible causes of unaccounted variability in other parameters. Patients 2 and 3 had slightly high blood pressure recordings. Patient 3 is on medication to help with his high blood pressure. Changes in blood pressure have potential implications on parameters investigated in this study, most notably, lumen diameter. To a certain point, higher blood pressures indicate larger lumen diameters, while low blood pressures indicate smaller lumen diameters. None of the patients exhibited large changes in blood pressure before and after the UBM examination.

TABLE XI

BLOOD PRESSURE RESULTS

\begin{tabular}{|l|l|}
\hline \multicolumn{2}{|c|}{ Patient 1 } \\
\hline BP Before & $116 / 68$ \\
\hline BP After & $128 / 70$ \\
\hline
\end{tabular}

\begin{tabular}{|l|l|}
\hline \multicolumn{2}{|c|}{ Patient 2 } \\
\hline BP Before & $142 / 82$ \\
\hline BP After & $150 / 82$ \\
\hline
\end{tabular}

\begin{tabular}{|l|l|}
\hline \multicolumn{2}{|c|}{ Patient 3 } \\
\hline BP before & $150 / 96$ \\
\hline BP after & $150 / 96$ \\
\hline
\end{tabular}

\begin{tabular}{|l|l|}
\hline \multicolumn{2}{|c|}{ Patient 4 } \\
\hline BP before & $120 / 80$ \\
\hline BP after & $128 / 88$ \\
\hline
\end{tabular}




\subsection{Oxygen saturation measurements}

The oxygen saturation measurements did not vary much between patients or between hands. Oxygen saturation is a relative measure of the amount of oxygen that is dissolved or carried in a given medium. The results indicate that the digits analyzed have about the same level of oxygenation. Since oxygen is carried in the blood stream, it is an indirect indication of perfusion. The results showed that these digits are receiving enough oxygen and are being adequately perfused. All measurements (Table XII) ranged between $94 \%$ and $98 \%$, with no significant differences noted. Oxygen saturations in the transplanted hand averaged $96.6 \%$ and those in the native hand averaged $96.4 \%$.

TABLE XII

OXYGEN SATURATION RESULTS

\begin{tabular}{|r|r|r|r|}
\hline & \multicolumn{3}{|c|}{ Transplant Oxygen saturation (\%) } \\
\hline \multicolumn{1}{|c|}{ Subject } & \multicolumn{1}{|c|}{ Digit 3 } & \multicolumn{1}{c|}{ Digit 4 } & \multicolumn{1}{l|}{ Digit 5 } \\
\hline 1 & 94 & 96 & 95 \\
\hline 2 & 97 & 96 & 96 \\
\hline 3 & 97 & 97 & 97 \\
\hline 4 & 98 & 98 & 98 \\
\hline
\end{tabular}

\begin{tabular}{|r|r|r|r|}
\hline & \multicolumn{3}{|c|}{ Native Oxygen saturation (\%) } \\
\hline Subject & \multicolumn{1}{|c|}{ Digit 3 } & \multicolumn{1}{|c|}{ Digit 4 } & \multicolumn{1}{l|}{ Digit 5 } \\
\hline 1 & 96 & 96 & 96 \\
\hline 2 & 97 & 96 & 95 \\
\hline 3 & 97 & 97 & 97 \\
\hline 4 & 97 & 96 & 97 \\
\hline
\end{tabular}

\subsection{Hoop stress calculation for arterial wall}

The hoop stress was found to be significantly $(\mathrm{p}=0.010)$ lower in the arteries of the transplanted hand. The average hoop stress (Table XIII) calculated for the 
transplanted hand was $52.46 \mathrm{kPa}$, while the hoop stress for the native hand was 81.08 $\mathrm{kPa}$. This conclusion is logical, considering that arteries in the transplanted hand have slightly lower pressures, smaller lumen diameters, and increased wall thicknesses. Lower pressures lead to lower hoop stress since the hoop stress is directly related to pressure. Similarly, smaller LD lead to smaller hoop stress values since it is also directly related to hoop stress. Furthermore, increased wall thicknesses lead to smaller hoop stress since wall thickness is inversely proportional to hoop stress. Combining these factors into the equation for hoop stress would result in a lower result. Increasing the wall thickness also increases the vessel stiffness. It is well documented that as people get older, their arteries tend to stiffen (Choi, 2010; Safar, 2000; Weber, 2004). It has also been observed that the progression of IH increases with age (Subbotin, 2007; Osika, 2007). These observations are related since increased IH results in thicker arterial walls, and thicker walls create stiffer vessels. Increased stiffness (caused by increased wall thickness) would have decreased hoop stress since hoop stress is inversely proportional to wall thickness. Thus, progressing IH should result in lower hoop stress values. 
TABLE XIII

HOOP STRESS RESULTS

\begin{tabular}{|c|c|c|c|c|c|c|c|}
\hline \multirow[b]{2}{*}{ Subject } & \multicolumn{7}{|c|}{ Transplant Hoop Stress (kPa) } \\
\hline & Ulnar & Radial & Digit 1 & Digit 2 & Digit 3 & Digit 4 & Digit 5 \\
\hline 1 & 62.76 & 49.06 & & & 13.38 & 17.51 & 16.96 \\
\hline 2 & 93.40 & 76.70 & 43.50 & 38.58 & 50.00 & 57.93 & 60.03 \\
\hline 3 & 94.83 & 78.56 & & 54.26 & 67.70 & 45.43 & 58.32 \\
\hline 4 & 39.91 & 84.09 & 44.48 & 48.12 & 52.31 & 33.37 & 30.35 \\
\hline Average & $72.7 \pm 26.4$ & $72.1 \pm 15.7$ & $44 \pm 0.7$ & $47 \pm 7.9$ & $45.8 \pm 23$ & $38.6 \pm 17.2$ & $41.4 \pm 21.2$ \\
\hline
\end{tabular}

\begin{tabular}{|c|c|c|c|c|c|c|c|}
\hline & \multicolumn{7}{|c|}{ Native Hoop Stress (kPa) } \\
\hline Subject & Ulnar & Radial & Digit 1 & Digit 2 & Digit 3 & Digit 4 & Digit 5 \\
\hline 1 & 78.72 & 75.02 & 35.3 & 43.57 & 44.26 & 50.05 & 69.96 \\
\hline 2 & 44.95 & 129.9 & 80.75 & 74.39 & 87.95 & 65.86 & 64.97 \\
\hline 3 & 160.3 & 153.00 & 146.91 & 122.51 & 104.71 & 144.01 & 105.51 \\
\hline 4 & 63.42 & 109.11 & 30.01 & 45.80 & 45.16 & 51.11 & 42.94 \\
\hline Average & $86.8 \pm 50.9$ & $116.8 \pm 33.1$ & $73.2 \pm 54.1$ & $71.6 \pm 36.7$ & $70.5 \pm 30.6$ & $77.8 \pm 44.8$ & $70.8 \pm 25.9$ \\
\hline
\end{tabular}




\section{$\underline{5.6 \text { Comparisons of intima and lumen diameter to brachial indices }}$}

\subsubsection{Comparison of IT and brachial index measurements}

The Pearson correlation coefficient for IT and brachial indices is $0.109(\mathrm{p}=0.438)$. Statistical significance was defined as a p-value less than 0.05 , indicating a significant relationship between the parameters tested. A Pearson correlation coefficient of +1 would indicate a perfect positive (increasing) linear relationship. A Pearson correlation coefficient of -1 would indicate a perfect negative (decreasing) linear relationship. A Pearson correlation coefficient of 0 would indicate that the variables are not correlated with one another.

The high p-value here suggests there is no significant relationship between IT and brachial index measurements. In fact, if there were a relationship between these parameters, the Pearson correlation coefficient would be negative. Since clinicians consider low brachial indices to indicate obstructions that could compromise flow, as with IH, a low brachial index was expected to correlate with a high IT. These results suggest that brachial index measurements should not be used to predict intima thicknesses in hand transplant patients. Since IT thicknesses are a direct indication of IH, brachial index measurements do not correlate with levels of arteriopathy in these patients. Figure 9 illustrates the lack of a significant relationship between IT and brachial index. 


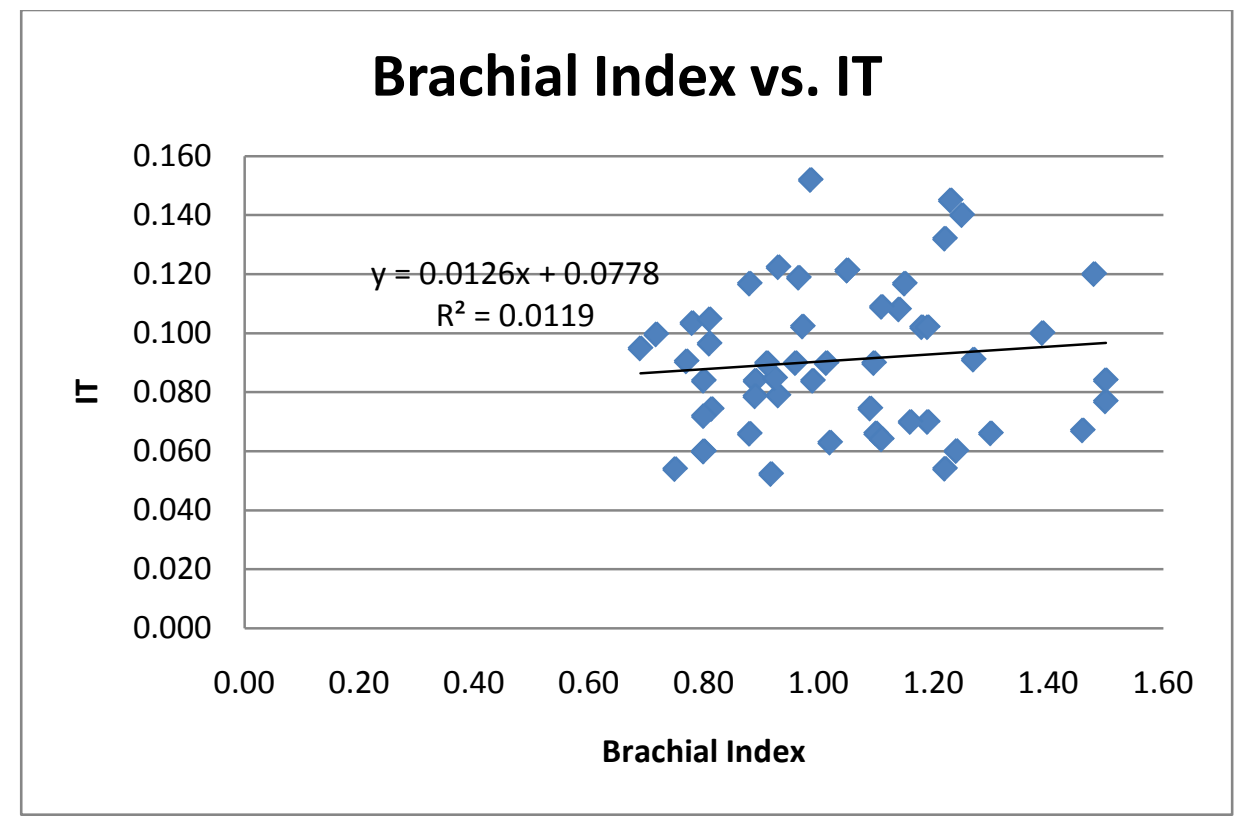

FIGURE 9 - Plot of brachial index vs. intima thickness

\subsubsection{Comparison of IT-to-lumen diameter and brachial index measurements}

The Pearson correlation coefficient between IT-to-lumen diameters and brachial index measurements was found to be $-0.368(\mathrm{p}=0.007)$. This $\mathrm{p}$-value indicates a significant relationship between IT-to-LD and brachial index. However, both the low correlation coefficient and examination of the plot of IT-to-LD (Figure 10) indicate that there is much variation and the regression line is a poor fit. Thus, brachial index measurements cannot reliably predict IT-to-lumen diameter ratios in hand transplant patients. This is valuable considering that both IT-to-lumen diameter and brachial indices are normalized values, which provides a more accurate comparison. Since IT-tolumen diameter is a measure of arteriopathy and the correlation between it and brachial index is not strong, brachial index measurements do not correlate with levels of arteriopathy. From the results here and from the correlation between IT and brachial index, hypotheses 3 and 4 are accepted. The sample size of this study was small; 
however, it included all of the remaining patients from the Louisville CTA program. The sample size could be increased by included any new hand transplant programs that are established in the United States. The results from this study suggest that the use of brachial indices to eliminate the suspicion of IH is invalid. In addition, since IH is such a strong indication of chronic rejection, the use of brachial indices to assess chronic rejection may also be invalid; a larger sample size is needed to confirm.

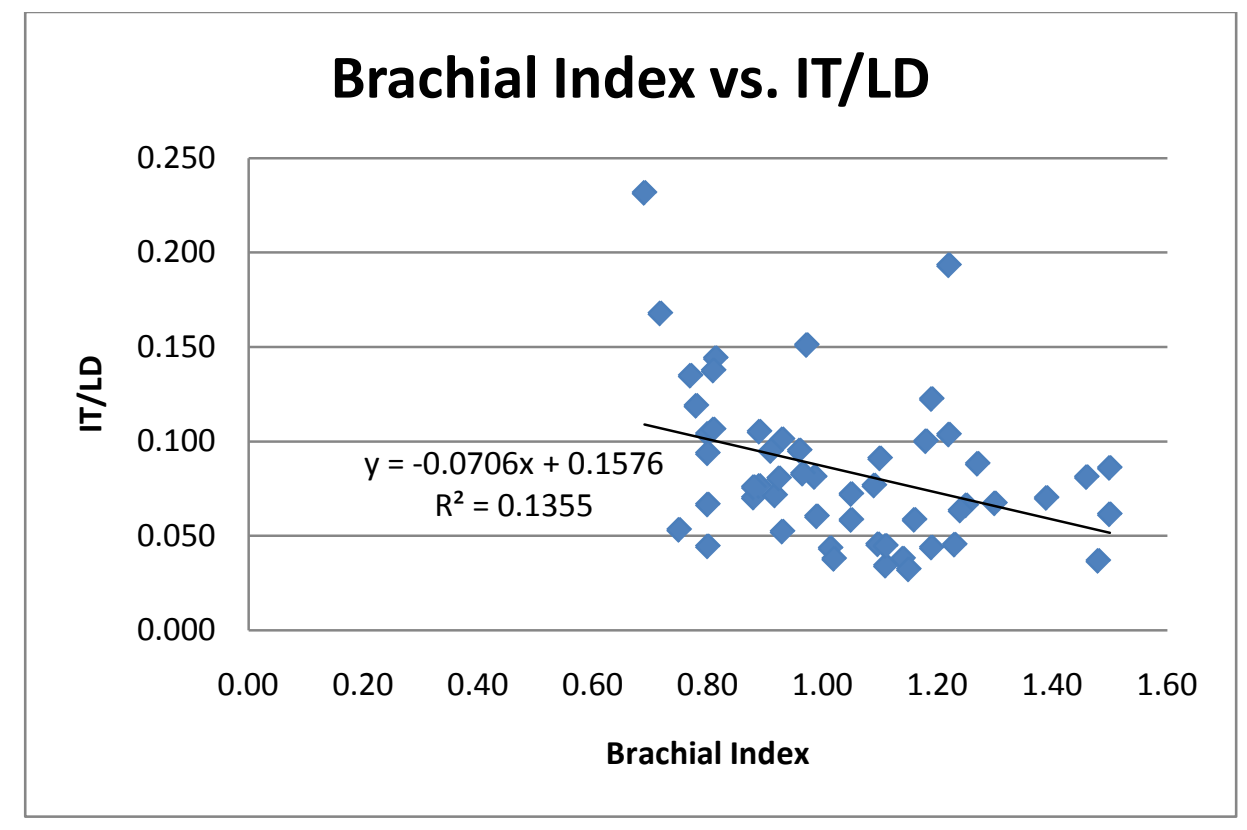

FIGURE 10 -- Plot of brachial index vs. IT-to-LD

\subsubsection{Comparison of IMT-to-lumen diameter and brachial index measurements}

The Pearson correlation coefficient between IMT-to-lumen diameters and brachial index measurements was found to be $-0.154(\mathrm{p}=0.271)$. Again, this high $\mathrm{p}$-value suggests that there is not a relationship between IMT-to-lumen diameter and brachial index. Thus, brachial index measurements cannot predict IMT-to-lumen diameter ratios in hand transplant patients. 


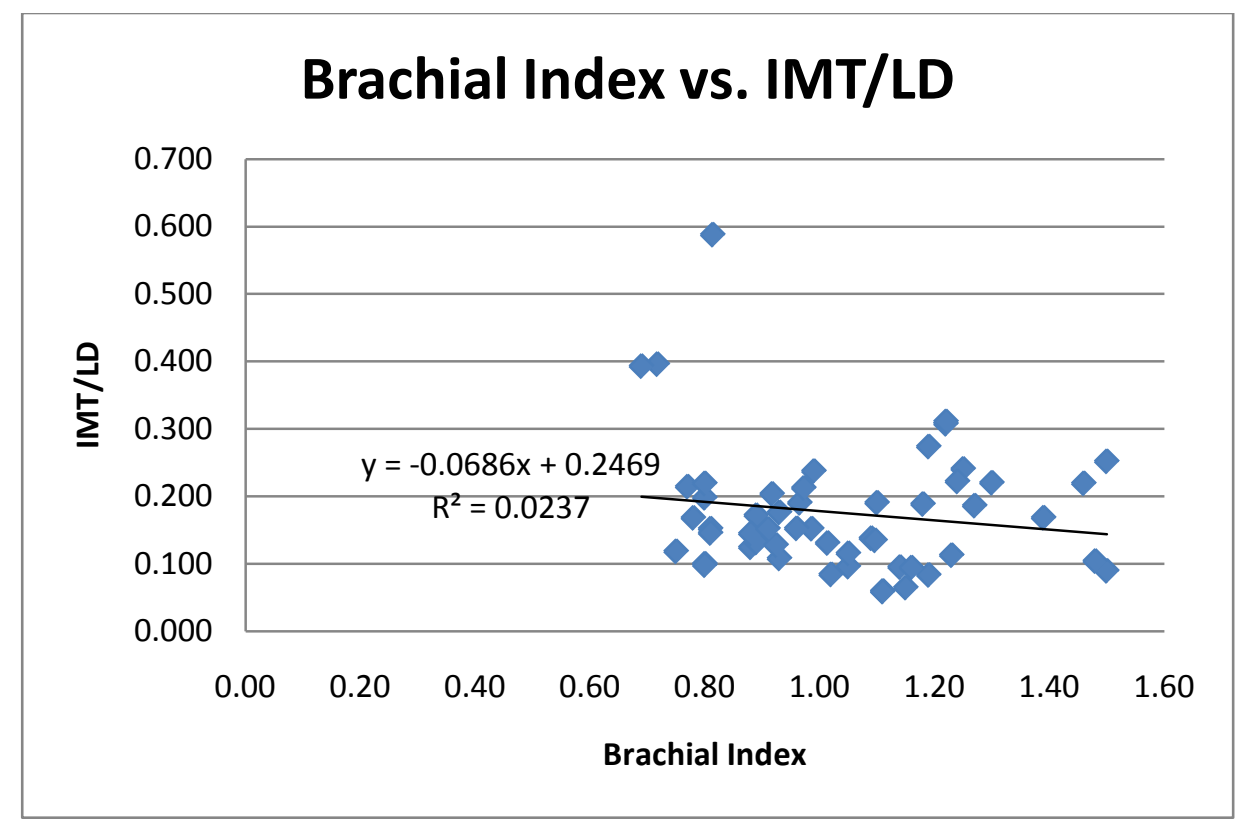

FIGURE 11 -- Plot of brachial index vs. IMT-to-LD 


\section{CONCLUSIONS}

The following conclusions have been established based upon the results of this study. Though this study included all available patients at the Louisville CTA program, increasing the sample the size of this study would be beneficial.

- Intima thicknesses are significantly larger in the transplanted arm than in the native arm in a hand transplant patient.

- The intima-to-lumen diameter ratios are significantly larger in the transplanted hand than in the native hand in a hand transplant patient.

- Hoop stress in the arterial wall is significantly lower in the transplanted hand than in the native hand of a hand transplant patient.

- The UBM provides an accurate, repeatable and real-time method to investigate the arteriopathy of a hand transplant patient.

- The UBM provides a way for clinicians to monitor the progression of arteriopathy in the hand, including intimal hyperplasia, over time. 


\section{RECOMMENDATIONS}

To take this project further, there are many recommendations, as there are still many unanswered questions. First, the UBM measurement protocol described here should be added to the remaining hand transplant patients' annual examination in the Louisville CTA Program. Future patients should start with an UBM examination pretransplantation and, if possible, the donated graft should also be examined either before or soon after transplantation. Due to inflammation and swelling after surgery, it may not be possible to perform an UBM examination until several weeks after the surgery. Nonetheless, it should be done in order to track changes in IT, IT-to-LD, IMT-to-LD and the overall arteriopathy over time to better understand chronic rejection and the mechanisms and/or factors associated with chronic rejection.

The pulse Doppler mode of the UBM must be investigated more closely. The author found that when examining an arterial section with the UBM machine, a cine loop of images could be obtained at one frequency, but a velocity waveform could not be detected. Thus, separate, careful, detailed velocity measurements need to be obtained to provide hemodynamic information. Velocity measurements are a better indicator of blood flow. The ultimate cause of amputation of a CTA is significantly reduced blood flow, velocity measurements are imperative to monitor. The velocity should be used as a better predictor of intimal hyperplasia. In addition, the UBM system should be characterized for humans and adjustments to the beam angle should be investigated to compare velocity measurements made in the same artery at the same time with different beam angles. 
Once the Doppler mode is better understood, the wall shear stress should be determined for each of the arteries included in this study. It has been shown that low shear stress, oscillating shear forces at the arterial floor and the heel of an anastomosis, as well as a high wall shear stress gradient at the toe of an anastomosis likely accelerate the IH growth (Haruguchi, 2003). The wall shear stress must be calculated at increments distally along the arterial tree utilizing a normalized length-to-diameter ratio for the respective artery under investigation to elucidate whether IT or IH is localized or distributed along the entire length of the artery. Using an MRI scan of the arms would facilitate this and provide the framework for a computation model of the patient's vasculature. The MRI will collect the anatomical data of the vascular structures. This data will be digitized into a CAD model and imported into a computational flow dynamics (CFD) software package to attain wall shear data using the velocity/flow and pressure data obtained from the UBM and Flo-Lab, which will be used as input parameters for the computational modeling.

Further improvements to the analysis methods could be incorporated by integrating an automation system to make the measurements. Finding a common anatomical location to start at, measurements could be taken at normalized increments downstream to the distal end of the artery. Data from different patients could be aligned to create a standard protocol for analysis. This would eliminate the error associated with intra-observer variability. In addition, it would create a more robust analysis of the data. This could be done for each patient and monitored over time.

An investigation of the parameters used in this project in a larger sample of clinically normal individuals should also be conducted to determine the variability of 
these parameters in a larger population, since small levels of intimal hyperplasia are normal. In addition, factors such as smoking, age, gender, and hand dominance can be explored to determine their effect on intimal hyperplasia. The intima thicknesses of the hand transplant patients can be compared to those of the normal population to better assess the level of arteriopathy that is present in these patients. 


\section{REFERENCES}

“Amputation Statistics by Cause: Limb Loss in the United States.” National Limb Loss Information Center. September 18, 2008. <http://www.amputeecoalition.org/fact_sheets/amp_stats_cause.html>. May 5, 2010.

Asakura T., Karino T. 1990. Flow patterns and spatial distribution of atherosclerotic lesions in human coronary arteries. Circulation Research 66:1045-1066.

Breidenbach, W., Gonzales, R., Kaufman, L., Klapheke, M., Tobin, G., Gorantla, V. 2008. Outcomes of the first 2 American hand transplants at 8 and 6 years posttransplant. Journal of Hand Surgery 33A:1039-1047.

Cebdales, L., Kanitakis, J., Schneeberger, S., Burns, C., Ruiz, P., Landin, L., Remmelink, M., Hewitt, C., Landgren, T., Lyons, B., Drachenberg, B., Solez, K., Kirk, A., Kleiner, D., Racusen, L. 2008. The Banff 2007 working classification of skincontaining composite tissue allograft pathology. American Journal of Transplantation 8:1396-1400.

Choi, C., Kim, E., Kim, S., Shin, S., Choi, U., Kim, J, Lim, H. Rha, S., Park, C. Seo, H., Oh, D. 2010. "Differing effects of aging on central and peripheral blood pressures and pulse wave velocity: a direct intraarterial study." Journal of Hypertension. 28.6 (2010):1252-1260.

Demetris, A., Murase, N., Starzl, T., Fung, J. 1998. Pathology of chronic rejection: an overview of common findings and observations about pathogenic mechanisms and possible prevention. Graft. 1:52-59.

Dubernard JM, Lengele B, Morelon E. 2007. Outcomes 18 months after the first human partial face transplantation. $N$ Engl J Med 357:2451.

Ferrara, J., Levine, J., Reddy, P., Holler, E. 2009. Graft-versus-host disease. Lancet. 373:1550-61.

Gelberman, R., Urbaniak, J., Bright, D., Levin, L. 1978. Digital sensibility following replantation. Journal of Hand Surgery 3:313-319.

Geraghty, J., Stoltenberg, R., Sollinger, H., Hullett, D. 1996. Vascular smooth muscle cells and neointimal hyperplasia in chronic transplant rejection. Transplantation 64:502-509.

Glagov, S. 1994. Intimal hyperplasia, vascular remodeling, and the restenosis problem. Circulation. 89: 2888-2891.

Gonzalez RN, Gorantla VS, Breidenbach WC. 2005. Complications after hand transplantation: Osteonecrosis of the hips [abstract]. World Society for Reconstructive Microsurgery (WSRM) III Congress, Part III. Buenos Aires, Argentina. 
Gross, W., Flanigan, D., Kraft, R., Stanley, J. 1978. Chronic upper extremity arterial insufficiency. Archives of Surgery 113:419-423.

Haruguchi, H., Teraoka, S. 2003. Intimal hyperplasia and hemodynamic factors in arterial bypass and arteriovenous grafts: a review. Japanese Society for Artificial Organs 6:227-235.

Hayry P, Mennander A, Tiisala S,. 1991. Rat aortic allografts: an experimental model for chronic transplant arteriosclerosis. Transplant Proc. 23: 611.

Johansson, M., Myredal, A., Friberg, P., Gan, L. 2010. High-resolution ultrasound showing increased intima and media thickness of the radial artery in patients with end-stage renal disease. Atherosclerosis doi:10.1016/j.atherosclerosis.2010.01.031.

Jones JW, Gruber SA, Barker JH, Breidenbach WC. 2000. Successful hand transplantation: One-year follow-up. Louisville Hand Transplant Team. New England Journal of Medicine 343:468-73.

Kaufman, C., Blair, B., Murphy, E., Breidenbach, W. 2009. A new option for amputees: Transplantation of the hand. Journal of Rehabilitation Research \& Development 46:395-404.

Keynton, R. 1995. The effect of graft caliber upon caliber upon hemodynamics and intimal hyperplasia in the distal anastomosis of chronic vascular bypass grafts. Ph.D diss., University of Akron Dissertation.

Keynton, R., Evancho, M., Sims, R., Rodway, N., Gobin, A., Rittgers, S. 2001. Intimal hyperplasia and wall shear in arterial bypass graft distal anastomoses: an in vivo model study. J Biomech Eng. 123(5):464-473.

Kohler, T., Jawein. A. 1992. Flow affects development of intimal hyperplasia after arterial injury in rats. Arteriosclerosis and Thrombosis. 12:963-971.

Merville, Pierre. 2005. Combating chronic renal allograft dysfunction. Drugs 65: 615631.

Osika, W., Dangardt, F., Gronos, J., Lundstam, U., Myredal, A., Johansson, M., Volkmann, R., Gustavsson, T., Gan, L., Friberg, P. 2007. Increasing peripheral artery thickness from childhood to seniority. Arteriosclerosis, Thrombosis, and Vascular Biology 27:671-676.

Petit, F., Minns, A., Dubernard, J., Hettiaratchy, S., Lee, A. 2003. Composite tissue allotransplantation and reconstructive surgery: first clinical applications. Annals of Surgery 237:19-25.

Petruzzo, P., Lanzetta, M., Dubernard, J., Margreiter, R., Schuind, F., Breidenbach, W., Nolli, R., Schneeberger, S., van Holder, C., Kaufman, C., Jablecki, J., Landin, L., 
Cavadas, P. 2008. The international registry on hand and composite tissue transplantation. Transplantation 86: 487-492.

Ravindra, Buell, Kaufman, Blair, Marvin, Nagubandi, Breidenbach, MD. 2008. Hand transplantation in the United States: Experience with 3 patients. Surgery 144(4):638-43.

Razuvaev, A., Lund, K., Roy, J., Hedin, U., Caidahl, K. 2008. Noninvasive real-time imaging of intima thickness after rat carotid artery balloon injury using ultrasound biomicroscopy. Atherosclerosis 199:310-316.

Ross R. 1993. Atherosclerosis: a defence mechanism gone awry. Am J Pathol. 143: 987.

Safar ME, London GM. 2000. Therapeutic studies and arterial stiffness in hypertension: recommendations of the European Society of Hypertension. J Hypertens. 18:1527-1535.

Shier, D., Butler, J., Lewis, R. 2006. Hole’s Essentials of Human Anatomy and Physiology. New York: McGraw-Hill.

Stary HC, Blankenhorn DH, Chandler AB, Glagov S, Insull W Jr, RichardsonM, Rosenfeld ME, Schaffer SA, Schwartz CJ, Wagner WD. 1992. A definition of the intima of human arteries and of its atherosclerosis-prone regions. A report from the Committee on Vascular Lesions of the Council on Arteriosclerosis. American Heart Association. Arterioscler Thromb.12:120-134.

Stone, James. 2004. Intimal hyperplasia in the distal ulnar artery: Influence of gender and implications for the hypothenar hammer syndrome. Cardiovascular Pathology 13:20-25.

Subbotin, Vladimir. 2007. Analysis of arterial intimal hyperplasia: review and hypothesis. Theoretical Biology and Medical Modelling 41:1-20.

Weber T, Auer J, O’Rourke M, Kvas E, Lassnig E, Berent R, Eber B. 2004. Arterial stiffness, wave reflections, and the risk of coronary artery disease. Circulation. 109:184-189.

Wendelhag, I., Gustavsson, T., Suurkula, M. 1991. Ultrasound measurement of wall thickness in the carotid artery: fundamental principles and description of a computerized analyzing system. Clin Physiol 11(6): 565-77.

Ziegler-Graham, K., MacKenzie, E., Ephraim, P., Travison, T., Brookmeyer, R. 2008. Estimating the Prevalence of Limb Loss in the United States - 2005 to 2050. Archives of Physical Medicine and Rehabilitation 89: 422-429.

Zimmerman, N., Zimmerman, S., McClinton, M., Wilgis, E., Koontz, C., Buehner, J. 1994. Long-term Recovery Following Surgical Treatment for Ulnar Artery Occlusion. The Journal of Hand Surgery 19A:17-21. 


\section{VITA}

Emily Renee Cox was born on March 11, 1987 in Rantoul, IL to Ricky Dale and Joan Carol Cox. She has one younger brother, David Christopher and two younger sisters, Jennifer Lauren and Olivia Nicole. She graduated from Holy Cross High School in 2005. She began her undergraduate studies at the University of Louisville in 2005. She completed three co-ops with DePuy Orthopaedics, Johnson \& Johnson in Warsaw, IN. In May 2009, she received her Bachelor of Science degree with highest honors in Bioengineering, as part of the first graduating class of the J.B. Speed School's Bioengineering program.

During her time at the University of Louisville, she was employed by the university tutoring service, Resources of Academic Achievement (REACH). She tutored engineering Calculus III, as well as Differential Equations. She was the manager of the Math Resource Center as a graduate student assistant. She was involved with the BioMedical Engineering Society (BMES). She was also involved with the engineering honors society, Tau Beta Pi, where she served as corresponding secretary.

Emily will return to the company that she completed her co-ops with, DePuy, as a Project Engineer in the Knee Surgical Technology teams. She plans on pursuing a Master of Business Administration after gaining a few years of working experience. 